\title{
Recent developments of Lyapunov-type inequalities for fractional differential equations
}

\author{
Sotiris K. Ntouyas, Bashir Ahmad and Theodoros P. Horikis
}

\begin{abstract}
A survey of results on Lyapunov-type inequalities for fractional differential equations associated with a variety of boundary conditions is presented. This includes Dirichlet, mixed, Robin, fractional, Sturm-Liouville, integral, nonlocal, multi-point, anti-periodic, conjugate, right-focal and impulsive conditions. Furthermore, our study includes Riemann-Liouville, Caputo, Hadamard, Prabhakar, Hilfer and conformable type fractional derivatives. Results for boundary value problems involving fractional $p$-Laplacian, fractional operators with nonsingular MittagLeffler kernels, $q$-difference, discrete, and impulsive equations, are also taken into account.
\end{abstract}

\section{Introduction and Preliminaries}

Integral inequalities are fundamental in the study of quantitative properties of solutions of differential and integral equations. The Lyapunov-type inequality is one of such inequalities when investigating the zeros of solutions of differential equations. A method for deriving a Lyapunov-type inequality for boundary value problems dates back to Nehari [1] and is based on the idea of converting the given problem into an integral equation. To illustrate this method, let us consider the following

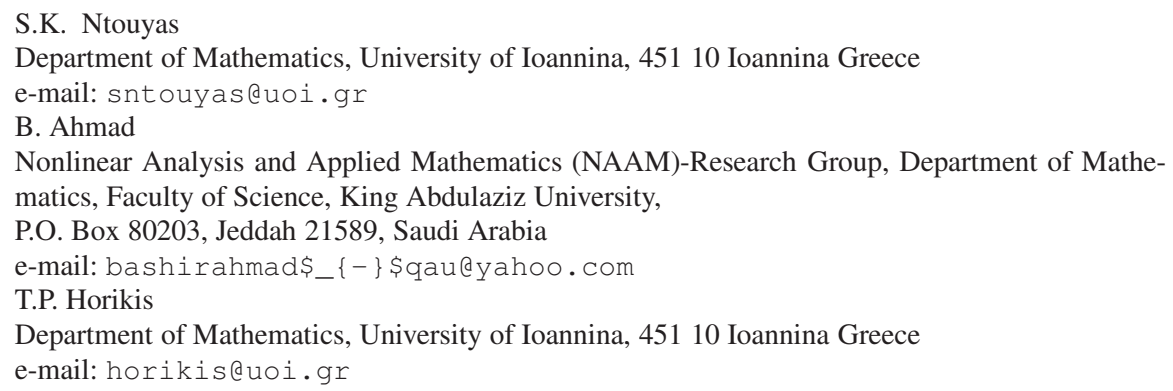


boundary value problem:

$$
\left\{\begin{array}{l}
y^{\prime \prime}(t)+q(t) y(t)=0, \quad a<t<b \\
y(a)=y(b)=0
\end{array}\right.
$$

where $a, b \in \mathbb{R}, a<b$ are consecutive zeros of $y(t)$ and $y(t) \not \equiv 0$, for all $t \in(a, b)$. It can easily be shown that problem (1) is equivalent to the integral equation

$$
y(t)=\int_{a}^{b} G(t, s) q(s) y(s) d s,
$$

where $G(t, s)$ is the Green's function given by

$$
G(t, s)=- \begin{cases}\frac{(t-a)(b-s)}{b-a}, & a \leq s \leq t \leq b \\ \frac{(s-a)(b-t)}{b-a}, & a \leq t \leq s \leq b .\end{cases}
$$

Taking the absolute value of both sides of equation (2), and taking into account that $y$ does not have any zeros in $(a, b)$,we get

$$
1 \leq \max _{a \leq t \leq b} \int_{a}^{b}|G(t, s)||q(s)| d s
$$

which yields the desired Lyapunov inequality

$$
\int_{a}^{b}|q(s)| d s \geq \frac{1}{\max _{(t, s) \in[a, b] \times[a, b]}|G(t, s)|} .
$$

In the special case where can find $H(s)$ explicily such that $\max _{t \in[a, b]}|G(t, s)| \leq H(s)$, then we obtain the following inequality

$$
1 \leq \int_{a}^{b} H(s)|q(s)| d s .
$$

Clearly the function $H(t)$ for problem (1) is $\frac{(t-a)(b-t)}{b-a}$. Moreover, if we take the absolute maximum of the function $H(t)$ for all $t \in[a, b]$, then it is obtained the following well-known Lyapunov inequality [2].

Theorem 1. If the boundary-value problem (1) has a nontrivial solution, where $q$ is a real and continuous function, then

$$
\int_{a}^{b}|q(s)| d s>\frac{4}{b-a} .
$$

The factor 4 in the above inequality is sharp and cannot be replaced by a larger number. 
Later Wintner in [3] and more authors thereafter generalized this result by replacing the function $|q(t)|$ in 6 by the function $q^{+}(t), q^{+}(t)=\max \{q(t), 0\}$, where now the resulting inequality reads:

$$
\int_{a}^{b} q^{+}(s) d s>\frac{4}{b-a}
$$

with $q^{+}(t)=\max \{q(t), 0\}$.

In [4], Hartman expanded further this result with the following inequality

$$
\int_{a}^{b}(b-t)(t-a) q^{+}(t) d t>(b-a)
$$

which is sharper than both (6) and (7).

Clearly, (8) implies (7) as $(b-t)(t-a) \leq \frac{(b-a)^{2}}{4}$ for all $t \in[a, b]$ and the equality holds when $t=\frac{a+b}{2}$.

It is worth mentioning that inequality (6) has found many practical applications in differential equations (oscillation theory, disconjugacy, eigenvalue problems, etc.), for instance, see [5]-[11] and references therein. A thorough literature review dealing with continuous and discrete Lyapunov inequalities and their applications can be found in [12] and [13] (which also includes an excellent account on the history of such inequalities).

In many engineering and scientific disciplines such as physics, chemistry, aerodynamics, electrodynamics of complex media, polymer rheology, economics, control theory, signal and image processing, biophysics, blood flow and related phenomena, fractional differential and integral equations represent processes in a more effective manner than their integer-order counterparts. This aspect has led to the increasing popularity in the study of fractional order differential and integral equations among mathematicians and researchers. In view of their extensive applications in various fields, the topic of inequalities for fractional differential equations has also attracted a significant attention in recent years.

This survey article is organized as follows. In Section 2 we introduce the reader to some basic concepts of fractional calculus. In Section 3 we summarize Lyapunovtype inequalities for fractional boundary value problems with different kinds of boundary conditions. In Section 4 we consider the inequalities for nonlocal and multi-point boundary value problems. Results on $p$-Laplacians are discussed in Section 5, while results on mixed fractional derivatives are given in Section 6. Section 7 deals with Lyapunov-type inequalities for Hadamard fractional differential equations. In Section 8, inequalities involving Prabhakar fractional differential equations are discussed. Section 9 contains the results on fractional $q$-difference equations, while Section 10 consists of the results involving fractional derivatives with respect to a certain function. Inequalities involving left and right derivatives, operators with nonsingular Mittag-Leffler kernels, discrete fractional differential equations, and impulsive fractional boundary value problems are respectively given in Sections 
$11,12,13$ and 14 respectively. We include the results for Hilfer rand Katugampola fractional differential equations in Sections 15 and 16 respectively, and conclude with Section 17 with results on conformable fractional differential equations. Note that our goal here is a more complete and comprehensive review and as such the choice is made to include as many results as possible to illustrate the progress on the matter. Any proofs (which are rather long) are omitted, for this matter, and the reader is referred to the relative article accordingly.

\section{Fractional Calculus}

Here we introduce some basic definitions of fractional calculus [14, 15] and recall some results that we need in the sequel.

Definition 1. (Riemann-Liouville fractional integral) Let $\alpha \geq 0$ and $f$ be a real function defined on $[a, b]$. The Riemann-Liouville fractional integral of order $\alpha$ is defined by $\left(I^{0} f\right)(x)=f(x)$ and

$$
\left(I^{\alpha} f\right)(t)=\frac{1}{\Gamma(\alpha)} \int_{a}^{t}(t-s)^{\alpha-1} f(s) d s, \quad \alpha>0, \quad t \in[a, b]
$$

provided the right hand side is point-wise defined on $[0, \infty)$, where $\Gamma(\alpha)$ is the Euler Gamma function: $\Gamma(\alpha)=\int_{0}^{\infty} t^{\alpha-1} e^{-t} d t$.

Definition 2. (Riemann-Liouville fractional derivative) The Riemann-Liouville fractional derivative of order $\alpha \geq 0$ is defined by $\left(D^{0} f\right)(t)=f(t)$ and

$$
\left(D^{\alpha} f\right)(t)=\left(D^{m} I^{m-\alpha} f\right)(t) \text { for } \alpha>0,
$$

where $m$ is the smallest integer greater than or equal to $\alpha$.

Definition 3. (Caputo fractional derivative) The Caputo fractional derivative of order $\alpha \geq 0$ is defined by $\left({ }^{C} D^{0} f\right)(t)=f(t)$ and

$$
\left({ }^{C} D^{\alpha} f\right)(t)=\left(I^{m-\alpha} D^{m} f\right)(t) \text { for } \alpha>0,
$$

where $m$ is the smallest integer greater than or equal to $\alpha$.

Notice that the differential operators of arbitrary order are nonlocal in nature and appear in the mathematical modeling of several real world phenomena due to this characteristic (see e.g. [14]).

If $f \in C([a, b], \mathbb{R})$, then the Riemann-Liouville fractional integral of order $\gamma>0$ exists on $[a, b]$. On the other hand, following [14, Lemma 2.2, p. 73], we know that the Riemann-Liouville fractional derivative of order $\gamma \in[n-1, n)$ exists almost everywhere (a.e.) on $[a, b]$ if $f \in A C^{n}([a, b], \mathbb{R})$, where $C^{k}([a, b], \mathbb{R})(k=$ $0,1, \ldots)$ denotes the set of $k$ times continuously differentiable mappings on $[a, b]$, 
$A C([a, b], \mathbb{R})$ is the space of functions which are absolutely continuous on $[a, b]$ and $A C^{(k)}([a, b], \mathbb{R})(k=1, \ldots)$ is the space of functions $f$ such that $f \in C^{k-1}([a, b], \mathbb{R})$ and $f^{(k-1)} \in A C([a, b], \mathbb{R})$. In particular, $A C([a, b], \mathbb{R})=A C^{1}([a, b], \mathbb{R})$. (We recall here that $A C([a, b], \mathbb{R})$ is the space of of functions $f$ which are absolutely continuous on $[a, b]$, and $A C^{n}([a, b], \mathbb{R})$ the space of functions $f$ which have continuous derivatives up to order $n-1$ on $[a, b]$ such that $\left.f^{(n-1)}(t) \in A C([a, b], \mathbb{R})\right)$.

Now we enlist some important results involving fractional order operators [14].

Proposition 1. Let $f$ be a continuous function on some interval $J$ and $p, q>0$. Then

$$
\left(I^{p} I^{q} f\right)(t)=\left(I^{p+q} f\right)(t)=\left(I^{q} I^{p} f\right)(t) \text { on } J .
$$

Proposition 2. Let $f$ be a continuous function on some interval I and $\alpha \geq 0$. Then

$$
\left(\mathscr{D}^{\alpha} I^{\alpha} f\right)(t)=f(t) \text { on } I,
$$

with $\mathscr{D}$ being the Riemann-Liouville or Caputo fractional derivative operator.

Proposition 3. The general solution of the following fractional differential equation

$$
\left(D^{q} y\right)(t)=f(t), t>a, 0<q \leq 1,
$$

is $y(t)=c(t-a)^{q-1}+\left(I^{q} f\right)(t), c \in \mathbb{R}$.

Proposition 4. The general solution of the following fractional differential equation

$$
\left({ }^{C} D^{q} y\right)(t)=f(t), t>a, 0<q \leq 1,
$$

is $y(t)=c+\left(I^{q} f\right)(t), c \in \mathbb{R}$.

\section{Lyapunov-type inequalities for fractional differential equations with different boundary conditions}

Lyapunov-type inequalities involving fractional differential operators have been investigated by many researchers in the recent years. In 2013, Ferreira [16] derived a Lyapunov-type inequality for Riemann-Liouville fractional differential equation with Dirichlet boundary conditions:

$$
\left\{\begin{array}{l}
D^{\alpha} y(t)+q(t) y(t)=0, \quad a<t<b, \\
y(a)=y(b)=0,
\end{array}\right.
$$

where $D^{\alpha}$ is the Riemann-Liouville fractional derivative of order $1<\alpha \leq 2$ and $q:[a, b] \rightarrow \mathbb{R}$ is a continuous function.

An appropriate approach for obtaining the Lyapunov inequality within the framework of fractional differential equations relies on the idea of converting the bound- 
ary value problem into an equivalent integral equation and then finding the maximum value of its kernel function (Green's function).

It is straightforword to show that the boundary value problem (9) is equivalent to the integral equation

$$
y(t)=\int_{a}^{b} G(t, s) q(s) y(s) d s,
$$

where $G(t, s)$ is the Green's function defined by

$$
G(t, s)=\frac{1}{\Gamma(\alpha)} \begin{cases}\frac{(b-s)^{\alpha-1}(t-a)^{\alpha-1}}{(b-a)^{\alpha-1}}, & a \leq t \leq s \leq b, \\ \frac{(b-s)^{\alpha-1}(t-a)^{\alpha-1}}{(b-a)^{\alpha-1}}-(t-s)^{\alpha-1}, & a \leq s \leq t \leq b .\end{cases}
$$

Observe that the Green's function (11) satisfies the following properties:

(i) $G(t, s) \geq 0, \forall t, s \in[a, b]$;

(ii) $\max _{s \in[a, b]} G(t, s)=G(s, s), s \in[a, b]$;

(iii) $G(s, s)$ has a unique maximum, given by

$$
\max _{s \in[a, b]} G(s, s)=G\left(\frac{a+b}{2}, \frac{a+b}{2}\right)=\frac{1}{\Gamma(\alpha)}\left(\frac{b-a}{4}\right)^{\alpha-1} .
$$

The Lyapunov inequality for problem (9) can be expressed as follows.

Theorem 2. If $y$ is a nontrivial solution of the boundary value problem (9), then

$$
\int_{a}^{b}|q(s)| d s>\Gamma(\alpha)\left(\frac{4}{b-a}\right)^{\alpha-1} .
$$

Here we remark that Lyapunov's standard inequality $\$ 6$ follows by taking $\alpha=2$ in the above inequality. Also, inequality (12) can be used to determine intervals for the real zeros of the Mittag-Leffler function:

$$
E_{\alpha}(z)=\sum_{k=1}^{\infty} \frac{z^{k}}{\Gamma(k \alpha+\alpha)}, z \in \mathbb{C}, \Re(\alpha)>0 .
$$

For the sake of convenience, let us consider the following fractional SturmLiouville eigenvalue problem (with $a=0$ and $b=1$ ):

$$
\left\{\begin{array}{l}
{ }^{C} D^{\alpha} y(t)+\lambda y(t)=0, \quad 0<t<1 \\
y(0)=y(1)=0
\end{array}\right.
$$

By Theorem 2 if $\lambda \in \mathbb{R}$ is an eigenvalue of (13), that is, if $\lambda$ is a zero of equation $E_{\alpha}(-\lambda)=0$, then $|\lambda|>\Gamma(\alpha) 4^{\alpha-1}$. Therefore the Mittag-Leffler function $E_{\alpha}(z)$ has no real zeros for $|z| \leq \Gamma(\alpha) 4^{\alpha-1}$. 
In 2014, Ferreira [17] replaced the Riemann-Liouville fractional derivative in problem (9) with Caputo fractional derivative ${ }^{C} D^{\alpha}$ and derived the following Lyapunovtype inequality for the resulting problem:

$$
\int_{a}^{b}|q(s)| d s>\frac{\Gamma(\alpha) \alpha^{\alpha}}{[(\alpha-1)(b-a)]^{\alpha-1}}
$$

In 2015, Jleli and Samet [18] considered the fractional differential equation

$$
{ }^{C} D^{\alpha} y(t)+q(t) y(t)=0, \quad 1<\alpha \leq 2, \quad a<t<b,
$$

associated with the mixed boundary conditions

$$
y(a)=0=y^{\prime}(b)
$$

or

$$
y^{\prime}(a)=0=y(b) .
$$

An integral equation equivalent to problem (15)-(16) is

$$
y(t)=\int_{a}^{b} G(t, s) q(s) y(s) d s,
$$

where $G(t, s)$ is again the Green's function defined by

$$
G(t, s)=\frac{H(t, s)}{\Gamma(\alpha)(b-s)^{2-\alpha}},
$$

and

$$
H(t, s)= \begin{cases}(\alpha-1)(t-\alpha), & a \leq t \leq s \leq b, \\ (\alpha-1)(t-\alpha)-(t-s)^{\alpha-1}(b-s)^{2-\alpha}, & a \leq s \leq t \leq b .\end{cases}
$$

The function $H$ satisfies the following inequality:

$$
|H(t, s)| \leq \max \{(2-\alpha)(b-s),(\alpha-1)(s-a)\} \text { for all }(t, s) \in[a, b] \times[a, b] .
$$
ity.

In relation to problem 15]-16, we have the following Lyapunov-type inequal-

Theorem 3. If y is a nontrivial solution of the boundary value problem (15)-(16), then

$$
\int_{a}^{b}(b-s)^{\alpha-2}|q(s)| d s>\frac{\Gamma(\alpha)}{\max \{\alpha-1,2-\alpha\}(b-a)} .
$$

In a similar manner, the Lyapunov-type inequality obtained for the boundary value problem (15)-17) is

$$
\int_{a}^{b}(b-s)^{\alpha-1}|q(s)| d s>\Gamma(\alpha) .
$$


As an application of Lyapunov-type inequalities (20) and 21), we can obtain the intervals, where certain Mittag-Leffler functions have no real zeros.

Corollary 1. Let $1<v \leq 2$. Then the Mittag-Leffler function $E_{\alpha}(z)$ has no real zeros for

$$
z \in\left(-\Gamma(\alpha) \frac{(\alpha-1)}{\max \{\alpha-1,2-\alpha\}}, 0\right] .
$$

The proof of the above corollary follows by applying inequality (20) to the following eigenvalue problem

$$
\left\{\begin{array}{l}
\left({ }^{C} D^{\alpha} y\right)(t)+\lambda y(t)=0, \quad 0<t<1, \\
y(0)=y^{\prime}(1)=0
\end{array}\right.
$$

Moreover, by applying inequality (21) to the following eigenvalue problem

$$
\left\{\begin{array}{l}
\left({ }^{C} D^{\alpha} y\right)(t)+\lambda y(t)=0, \quad 0<t<1, \\
y^{\prime}(0)=y(1)=0
\end{array}\right.
$$

we can obtain the following result:

Corollary 2. Let $1<v \leq 2$. Then the Mittag-Leffler function $E_{\alpha}(z)$ has no real zeros for

$$
z \in(\alpha \Gamma(\alpha), 0] .
$$

In 2015, Jleli et al. [19] obtained a Lyapunov-type inequality:

$$
\int_{a}^{b}(b-s)^{\alpha-2}(b-s+\alpha-1)|q(s)| d s \geq \frac{(b-a+2) \Gamma(\alpha)}{\max \{\mathrm{b}-\mathrm{a}+1,((2-\alpha) /(\alpha-1))(\mathrm{b}-\mathrm{a})-1\}},
$$

for the following problem with Robin boundary conditions

$$
\left\{\begin{array}{l}
{ }^{C} D^{\alpha} y(t)+q(t) y(t)=0,1<\alpha \leq 2, \quad a<t<b, \\
y(a)-y^{\prime}(a)=y(b)+y^{\prime}(b)=0
\end{array}\right.
$$

where $q:[a, b] \rightarrow \mathbb{R}$ is a continuous function.

Using the Lyapunov-type inequality 24], we can find an interval, where a linear combination of Mittag-Leffler functions $E_{\alpha, \beta}=\sum_{k=0}^{\infty} \frac{z^{k}}{\Gamma(k \alpha+\beta)}, \alpha>0, \beta>0, z \in$ $\mathbb{C}$ has no real zeros. In precise terms, we have the following result.

Theorem 4. Let $a<\alpha \leq 2$. Then $E_{\alpha, 2}(z)+E_{\alpha, 1}(z)+z E_{\alpha, \alpha}(z)$ has no real zeros for

$$
z \in\left(\frac{-3 \alpha \Gamma(\alpha)}{(1+\alpha) \max \{2,((2-\alpha) /(\alpha-1)-1\}}, 0\right] .
$$

In 2015, Rong and Bai [20] considered a boundary value problems with fractional boundary conditions: 


$$
\left\{\begin{array}{l}
{ }^{C} D^{\alpha} y(t)+q(t) y(t)=0,1<\alpha \leq 2, \quad a<t<b \\
y(a)=0,{ }^{C} D^{\beta} y(b)=0,0<\beta \leq 1
\end{array}\right.
$$

where $q:[a, b] \rightarrow \mathbb{R}$ is a continuous function. The Lyapunov-type inequality derived for problem (26) is

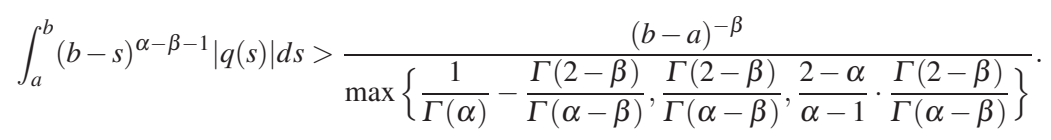

Later, Jleli and Samet [21] obtained a Lyapunov-type inequality for a boundary value problems with Sturm-Liouville boundary conditions

$$
\left\{\begin{array}{l}
{ }^{C} D^{\alpha} y(t)+q(t) y(t)=0,1<\alpha \leq 2, \quad a<t<b \\
p y(a)-r y^{\prime}(a)=y(b)=0, p>0, r \geq 0
\end{array}\right.
$$

where $q:[a, b] \rightarrow \mathbb{R}$ is a continuous function. The integral equation equivalent to the problem (28) is

$$
y(t)=\int_{a}^{b} G(t, s) q(s) y(s) d s,
$$

where $G(t, s)$ is the Green's function defined by

$$
G(t, s)=\frac{1}{\Gamma(\alpha)} \begin{cases}\frac{(r / p+t-a)}{r / p+b-a}(b-s)^{\alpha-1}, & a \leq t \leq s \leq b, \\ \frac{(r / p+t-a)}{r / p+b-a}(b-s)^{\alpha-1}-(t-s)^{\alpha-1}, & a \leq s \leq t \leq b .\end{cases}
$$

In order to estimate this Green's function, we distinguish two cases:

(i) If $\frac{r}{p}>\frac{b-a}{\alpha-1}$, then $0 \leq G(t, s) \leq G(s, s), \quad(t, s) \in[a, b] \times[a, b]$ with

$$
\max _{s \in[a, b]} G(t, s)=\frac{1}{\Gamma(\alpha)} \frac{(r / p)(b-a)^{\alpha-1}}{(r / p+b-a)} .
$$

(ii) If $0 \leq \frac{r}{p} \leq \frac{b-a}{\alpha-1}$, then $\Gamma(\alpha) G(t, s) \leq \max \{\mathscr{A}(\alpha, r / p), \mathscr{B}(\alpha, r / p)\}$, where

$$
\begin{gathered}
\mathscr{A}(\alpha, r / p)=\frac{(b-a)^{\alpha-1}}{(r / p+b-a)}\left(\left(\frac{(b-a)^{\alpha-1}}{(r / p+b-a)(\alpha-1)^{\alpha-1}}\right)^{\frac{1}{\alpha-2}}(2-\alpha)-\frac{r}{p}\right), \\
\mathscr{B}(\alpha, r / p)=\left(\frac{r}{p}+b-a\right)^{\alpha-1} \frac{(\alpha-1) \alpha-1}{\alpha^{\alpha}} .
\end{gathered}
$$

The Lyapunov inequalities corresponding to the above cases are given in the following result. 
Theorem 5. If there exists a nontrivial continuous solution of the fractional boundary value problem (28), then

$$
\begin{gathered}
\text { (i) } \int_{a}^{b}|q(s)| d s>\left(1+\frac{p}{r}(b-a)\right) \frac{\Gamma(\alpha)}{(b-a)^{\alpha-1}} \text { when } p>0, \frac{r}{p}>\frac{b-a}{\alpha-1} \\
\text { (ii) } \int_{a}^{b}|q(s)| d s>\frac{\Gamma(\alpha)}{\max \{\mathscr{A}(\alpha, r / p), \mathscr{B}(\alpha, r / p)\}} \text { when } p>0,0 \leq \frac{r}{p} \leq \frac{b-a}{\alpha-1}
\end{gathered}
$$

Using the above Lyapunov-type inequalities, we can find intervals, where linear combinations of some Mittag-Leffler functions have no real zeros.

Corollary 3. Let $1<\alpha<2, p>0, \frac{r}{p}>\frac{1}{\alpha-1}$. Then the linear combination of Mittag-Leffler functions given by

$$
p E_{\alpha, 2}(z)+q r E_{\alpha, 1}(z)
$$

has no real zeros for

$$
z \in\left(-\left(1+\frac{p}{r}\right) \Gamma(\alpha), 0\right]
$$

This corollary can be established by considering the following fractional SturmLiouville eigenvalue problem:

$$
\left\{\begin{array}{l}
{ }^{C} D^{\alpha} y(t)+\lambda y(t)=0, \quad 0<t<1, \\
p y(0)-r y^{\prime}(0)=y(1)=0 .
\end{array}\right.
$$

We can apply the foregoing Lyapunov-type inequalities to study the nonexistence of solutions for certain fractional boundary value problems. For example, the problem (28) with $p=1, r=2, a=0, b=1,3 / 2<\alpha<2$, has no nontrivial solution if $\int_{0}^{1}|q(s)| d s<\frac{3}{2} \Gamma(\alpha)$. As a second example, there is no nontrivial solution for the problem (28) with $p=2, r=1, a=0, b=1,1<\alpha<2$, provided that

$$
\int_{0}^{1}|q(s)| d s<\frac{\Gamma(\alpha)}{\max \{\mathscr{A}(\alpha, 1 / 2), \mathscr{B}(\alpha, 1 / 2)\}} .
$$

In 2015, O'Regan and Samet [22] obtained a Lyapunov-type inequality for the fractional boundary value problem:

$$
\left\{\begin{array}{l}
D^{\alpha} y(t)+q(t) y(t)=0, \quad a<t<b, \\
y(a)=y^{\prime}(a)=y^{\prime \prime}(a)=y^{\prime \prime}(b)=0,
\end{array}\right.
$$

where $D^{\alpha}$ is the standard Riemann-Liouville fractional derivative of fractional order $3<\alpha \leq 4$ and $q:[a, b] \rightarrow \mathbb{R}$ is a continuous function.

The integral equation associated with problem 33 is

$$
y(t)=\int_{a}^{b} G(t, s) q(s) y(s) d s
$$


where $G(t, s)$ is the Green's function defined by

$$
G(t, s)=\frac{1}{\Gamma(\alpha)} \begin{cases}\frac{(t-a)^{\alpha-1}(b-s)^{\alpha-3}}{(b-a)^{\alpha-3}}, & a \leq t \leq s \leq b, \\ \frac{(t-a)^{\alpha-1}(b-s)^{\alpha-3}}{(b-a)^{\alpha-3}}-(t-s)^{\alpha-1}, & a \leq s \leq t \leq b .\end{cases}
$$

The Green's function defined in (35) satisfies the following inequality:

$$
0 \leq G(t, s) \leq G(b, s)=\frac{(b-s)^{\alpha-3}(s-a)(2 b-a-s)}{\Gamma(\alpha)}, \quad(t, s) \in[a, b] \times[a, b] .
$$

The Lyapunov inequality for the problem (33) is as follows.

Theorem 6. If there exists a nontrivial continuous solution of the fractional boundary value problem (33), then

$$
\int_{a}^{b}(b-s)^{\alpha-3}(s-a)(2 b-a-s)|q(s)| d s \geq \Gamma(\alpha) .
$$

To demonstrate an application of the above inequality, we consider the eigenvalue problem:

$$
\left\{\begin{array}{l}
D^{\alpha} y(t)+\lambda y(t)=0, \quad 0<t<1,3<\alpha \leq 4 \\
y(0)=y^{\prime}(0)=y^{\prime \prime}(0)=y^{\prime \prime}(1)=0
\end{array}\right.
$$

Corollary 4. If $\lambda$ is an eigenvalue of the problem (37), then

$$
|\lambda| \geq \frac{\Gamma(\alpha)}{2 B(2, \alpha-2)}
$$

where $B$ is the beta function defined by $B(x, y)=\int_{0}^{1} s^{x-1}(1-s)^{y-1} d s, x, y>0$.

Sitho et al. [23] established some Lyapunov-type inequalities for the following hybrid fractional boundary value problem

$$
\left\{\begin{array}{l}
D_{a}^{\alpha}\left[\frac{y(t)}{f(t, y(t))}-\sum_{i=1}^{n} I_{a}^{\beta} h_{i}(t, y(t))\right]+g(t) y(t)=0, \quad t \in(a, b), \\
y(a)=y^{\prime}(a)=y(b)=0
\end{array}\right.
$$

where $D_{a}^{\alpha}$ denotes the Riemann-Liouville fractional derivative of order $\alpha \in(2,3]$ starting from a point $a$, the functions $g \in L^{1}((a, b], \mathbb{R}), f \in C^{1}([a, b] \times \mathbb{R}, \mathbb{R} \backslash\{0\})$, $h_{i} \in C([a, b] \times \mathbb{R}, \mathbb{R}), \forall i=1,2, \ldots, n$ and $I_{a}^{\beta}$ is the Riemann-Liouville fractional integral of order $\beta \geq \alpha$ with the lower limit at the point $a$. We consider two cases: (I) $h_{i}=0, i=1,2, \ldots, n$ and (II) $h_{i} \neq 0, i=1,2, \ldots, n$. 
Case I: $h_{i}=0, i=1,2, \ldots, n$. We consider the problem (38) with $h_{i}(t, \cdot)=0$ for all $t \in[a, b]$. For $\alpha \in(2,3]$, we first construct the Green's function for the following boundary value problem

$$
\left\{\begin{array}{l}
D_{a}^{\alpha}\left[\frac{y(t)}{f(t, y(t))}\right]+g(t) y(t)=0, \quad t \in(a, b), \\
y(a)=y^{\prime}(a)=y(b)=0
\end{array}\right.
$$

with the assumption that $f$ is continuously differentiable and $f(t, y(t)) \neq 0$ for all $t \in[a, b]$.

Let $y \in A C([a, b], \mathbb{R})$ be a solution of the problem $[39]$. Then the function $y$ satisfies the following integral equation

$$
y=f(t, y) \int_{a}^{b} G(t, s) g(s) y(s) d s,
$$

where $G(t, s)$ is the Green's function defined by (11) and satisfies the following properties:

(i) $G(t, s) \geq 0, \forall t, s \in[a, b]$.

(ii) $G(t, s) \leq H(s):=\frac{(b-s)^{\alpha-1}}{\Gamma(\alpha-1)}$.

(iii) $\max _{s \in[a, b]} H(s)=\frac{(b-a)^{\alpha-1}}{\Gamma(\alpha-1)}$.

Theorem 7. The necessary condition for existence of a nontrivial solution for the boundary value problem (39) is

$$
\frac{\Gamma(\alpha-1)}{\|f\|} \leq \int_{a}^{b}(b-s)^{\alpha-1}|g(s)| d s,
$$

where $\|f\|=\sup _{t \in[a, b], y \in \mathbb{R}}|f(t, y)|$.

Case II: $h_{i} \neq 0, i=1,2, \ldots, n$.

Let $y \in A C[a, b]$ be a solution of the problem $[38$. Then the function $y$ can be written as

$$
y(t)=f(t, y(t))\left[\int_{a}^{b} G(t, s) g(s) y(s) d s-\sum_{i=1}^{n} \int_{a}^{b} G^{*}(t, s) h_{i}(s, y(s)) d s\right],
$$

where $G(t, s)$ is defined as in (11) and $G^{*}(t, s)$ is defined by

$$
G^{*}(t, s)=\frac{1}{\Gamma(\beta)} \begin{cases}\frac{(b-s)^{\beta-1}(t-a)^{\alpha-1}}{(b-a)^{\alpha-1}}-(t-s)^{\beta-1}, & a \leq s \leq t \leq b, \\ \frac{(b-s)^{\beta-1}(t-a)^{\alpha-1}}{(b-a)^{\alpha-1}}, & a \leq t \leq s \leq b .\end{cases}
$$


The Green's function $G^{*}(t, s)$, which is given by [43), satisfies the following inequalities:

(i) $G^{*}(t, s) \geq 0, \quad \forall t, s \in[a, b]$;

(ii) $G^{*}(t, s) \leq J(s):=\frac{(\alpha-1)(b-s)^{\beta-1}}{\Gamma(\beta)}$.

Also we have

(iii) $\max _{s \in[a, b]} J(s)=\frac{(\alpha-1)(b-a)^{\beta-1}}{\Gamma(\beta)}$.

Theorem 8. Assume that $\left|h_{i}(t, y(t))\right| \leq\left|x_{i}(t)\right||y(t)|$, where $x_{i} \in C([a, b], \mathbb{R}), i=$ $1,2, \ldots, n$ and $[a, b]=[0,1]$. The necessary condition for existence of a nontrivial solution for the problem $(38)$ on $[0,1]$ is

$$
\Gamma(\alpha-1)\left(\frac{1}{\|f\|}-\frac{(\alpha-1)}{\Gamma(\beta+1)} \sum_{i=1}^{n}\left\|x_{i}\right\|\right) \leq \int_{0}^{1}(1-s)^{\alpha-1}|g(s)| d s .
$$

In 2016, Al-Qurashi and Ragoub [24] obtained a Lyapunov-type inequality for a boundary value problem with integral boundary condition

$$
\left\{\begin{array}{l}
{ }^{C} D^{\alpha} y(t)+q(t) y(t)=0, \quad a<t<b, \\
y(a)+\mu \int_{a}^{b} y(s) q(s) d s=y(b)
\end{array}\right.
$$

where ${ }^{C} D^{\alpha}$ is the Caputo fractional derivative of order $0<\alpha \leq 1, q:[a, b] \rightarrow \mathbb{R}$ is a continuous function, $a, b$ are consecutive zeros of the solution $y$ and $\mu$ is positive.

The function $y \in C([a, b], \mathbb{R})$ is a solution of the boundary value problem 445) if and only if $y$ satisfies the integral equation

$$
y(t)=\int_{a}^{b} G(t, s) q(s) y(s) d s,
$$

where $G(t, s)$ is the Green's function defined by

$$
G(t, s)=\frac{1}{\Gamma(\alpha)} \begin{cases}\frac{(b-s)^{\alpha-1}}{\mu(b-a)}-\frac{(b-s)^{\alpha}}{(b-a) \alpha}, & a \leq t \leq s \leq b, \\ \frac{(b-s)^{\alpha-1}}{\mu(b-a)}-\frac{(b-s)^{\alpha}}{(b-a) \alpha}-(t-s)^{\alpha-1}, & a \leq s \leq t \leq b,\end{cases}
$$

and satisfies the following properties:

(i) $G(t, s) \geq 0$, for all $a \leq t, s \leq b$;

(ii) $\max _{t \in[a, b]} G(t, s)=G(b, s), s \in[a, b]$; 
(iii) $G(b, s)$ has a unique maximum given by $\max _{s \in[a, b]} G(b, s)=\frac{\alpha(b-a+\mu)(\alpha \mu+1)^{\alpha-1}}{\Gamma(\alpha) \mu^{\alpha}(b-a)}$, provident $0<\mu(b-a)<\alpha$.

We describe the Lyapunov's inequality for the problem (45) as follows.

Theorem 9. The boundary value problem (45) has a nontrivial solution provided that the real and continuous function $q$ satisfies the following inequality

$$
\int_{a}^{b}|q(s)| d s>\frac{\Gamma(\alpha) \mu^{\alpha}(b-a)}{\alpha(b-a+\mu)(\alpha \mu+1)^{\alpha-1}} .
$$

In 2016, Fereira [25] obtained a Lyapunov-type inequality for a sequential fractional boundary value problem

$$
\left\{\begin{array}{l}
\left(D^{\alpha} D^{\beta} y\right)(t)+q(t) y(t)=0, \quad a<t<b, \\
y(a)=y(b)=0,
\end{array}\right.
$$

where $D^{\delta}, \delta=\alpha, \beta$ stands for the Riemann- Liouville fractional derivative and $q:[a, b] \rightarrow \mathbb{R}$ is a continuous function. Assuming that 49 has a nontrivial solution $y \in C[a, b]$ of the form

$$
y(t)=c \frac{\Gamma(\alpha)}{\Gamma(\alpha+\beta)}(t-a)^{\alpha+\beta-1}-\left(I^{\alpha+\beta} q y\right)(t),
$$

it follows by Proposition 2.6 and the fact $I^{\beta}(t-a)^{\alpha-1}=\frac{\Gamma(\alpha)}{\Gamma(\alpha+\beta)}(t-a)^{\alpha+\beta-1}$ that $y^{\prime}$ is integrable in $[a, b]$. Then, as argued in ([14, Section 2.3.6-2.3.7]), we have

$$
\left(D^{\alpha} D^{\beta} y\right)(t)=\left(D^{\alpha+\beta} y\right)(t) .
$$

The following result is therefore an immediate consequence of Theorem 2

Theorem 10. (Riemmann-Liouville Case) Let $0<\alpha, \beta \leq 1$ with $1<\alpha+\beta \leq 2$. If there exists a nontrivial continuous solution of the fractional boundary value problem (49), then

$$
\int_{a}^{b}|q(s)| d s>\Gamma(\alpha+\beta)\left(\frac{4}{b-a}\right)^{\alpha+\beta-1} .
$$

As an application we consider the following sequential fractional differential equation

$$
\left(D^{\alpha} D^{\alpha} y\right)(t)+\lambda^{2} y(t)=0, \quad \lambda \in \mathbb{R}, \quad t \in(0,1), \frac{1}{2}<\alpha \leq 1 .
$$

The fundamental set of solutions to [51) is

$$
\left\{\cos _{\alpha}(\lambda t), \sin _{\alpha}(\lambda t)\right\}
$$

where 


$$
\cos _{\alpha}(\lambda t)=\sum_{j=0}^{\infty}(-1)^{j} \lambda^{2 j} \frac{t^{(2 j+1) \alpha-1}}{\Gamma((2 j+1) \alpha)} \text { and } \sin _{\alpha}(\lambda t)=\sum_{j=0}^{\infty}(-1)^{j} \lambda^{2 j+1} \frac{t^{(j+1) 2 \alpha-1}}{\Gamma((j+1) 2 \alpha)} .
$$

Therefore the general solution of 51 can be written as

$$
y(t)=c_{1} \cos \alpha(\lambda t)+c_{2} \sin _{\alpha}(\lambda t), \quad c_{1}, c_{2} \in \mathbb{R} .
$$

Now, the nontrivial solutions of (51) for which the boundary conditions $y(0)=0=$ $y(1)$ hold, satisfy $\sin _{\alpha}(\lambda)=0$, where $\lambda$ is a real number different from zero (the eigenvalue of the problem). By Theorem 10 , the following inequality then holds

$$
\lambda^{2}>\Gamma(2 \alpha) 4^{2 \alpha-1}
$$

which can alternatively be expressed in form of the following result.

Corollary 5. Let $\frac{1}{2}<\alpha \leq 1$. If

$$
|t| \leq \sqrt{\Gamma(2 \alpha) 4^{2 \alpha-1}}, \quad t \neq 0
$$

then $\sin _{\alpha}(t)$ has no real zeros.

In [25], Fereira replaced the Riemmann-Liouville fractional derivative in the problem 49) with the Caputo fractional derivative and obtained the following Lyapunov-type inequality:

$$
\int_{a}^{b}|q(s)| d s>\frac{\Gamma(\alpha+\beta)}{(b-a)^{\alpha+\beta-1}} \frac{(\alpha+2 \beta-1)^{\alpha+2 \beta-1}}{(\alpha+\beta-1)^{\alpha+\beta-1} \beta^{\beta}} .
$$

In 2016, Al-Qurashi and Ragoub [26] obtained a Lyapunov-type inequality for a fractional boundary value problem:

$$
\left\{\begin{array}{l}
D^{\alpha} y(t)+q(t) y(t)=0, \quad 1<t<e, \\
y(a)=y(b)=y^{\prime \prime}(a)=y^{\prime \prime}(b)=0
\end{array}\right.
$$

where $D^{\alpha}$ is the standard Riemann-Liouville fractional derivative of order $3<\alpha \leq 4$ and $q:[a, b] \rightarrow \mathbb{R}$ is a continuous function.

The function $y \in C([a, b], \mathbb{R})$ is a solution of the boundary value problem $[53]$ if and only if $y$ satisfies the integral equation

$$
y(t)=\int_{a}^{b} G(t, s) q(s) y(s) d s,
$$

where $G(t, s)$ is the Green's function defined by 


$$
G(t, s)=\frac{1}{\Gamma(\alpha)} \begin{cases}-(t-a)(b-s)^{\alpha-1} \\ +\frac{\alpha(\alpha-1)(b-a)(b-s)^{\alpha-3}}{6}(t-a)\left[1-\frac{(t-a)^{2}}{(b-a)^{2}}\right], \quad a \leq t \leq s \leq b, \\ (t-s)^{\alpha-1}-(t-a)(b-s)^{\alpha-1} \\ +\frac{\alpha(\alpha-1)(b-a)(b-s)^{\alpha-3}}{6}(t-a)\left[1-\frac{(t-a)^{2}}{(b-a)^{2}}\right], \quad a \leq s \leq t \leq b,\end{cases}
$$

and satisfies the relation:

$$
0 \leq G(t, s) \leq G(b, s)=\frac{(1-(b-a))(b-a)^{\alpha-1}}{\Gamma(\alpha)}, \quad(t, s) \in[a, b] \times[a, b] .
$$

The Lyapunov-type inequality for the problem (53) is given in the following result.

Theorem 11. If there exists a nontrivial continuous solution to the fractional boundary value problem (53), then

$$
\int_{a}^{b}|q(s)| d s>\frac{\Gamma(\alpha)}{(1-(b-a))(b-a)^{\alpha-1}} .
$$

In order to illustrate Theorem 11, we apply the Lyapunov-type inequality (56) to find a bound for $\lambda$ so that the following eigenvalue problem has a nontrivial solution:

$$
\left\{\begin{array}{l}
D^{\alpha} y(t)+\lambda y(t)=0, \quad 0<t<\frac{1}{2}, 3<\alpha \leq 4 \\
y(1)=y\left(\frac{1}{2}\right)=y^{\prime \prime}(1)=y^{\prime \prime}\left(\frac{1}{2}\right)=0
\end{array}\right.
$$

Corollary 6. If $\lambda$ is an eigenvalue of the fractional boundary value problem (57), then the following inequality holds

$$
|\lambda| \geq \frac{\Gamma(\alpha)}{2^{-\alpha}}
$$

In 2016, Dhar et al. [27] derived Lyapunov-type inequalities for the following boundary value problem

$$
\left\{\begin{array}{l}
D^{\alpha} y(t)+q(t) y(t)=0, \quad a<t<b, 1<\alpha \leq 2 \\
D^{\alpha-2} y(a)=D^{\alpha-2} y(b)=0
\end{array}\right.
$$

where $D^{\alpha}$ is the Riemann-Liouville fractional derivative of order $\alpha(1<\alpha \leq 2)$, $q \in L([a, b], \mathbb{R})$. Their main result on fractional Lyapunov-type inequalities is the following.

Theorem 12. (a) If the problem (58) has a nontrivial solution, then 


$$
\max _{t \in[a, b]}\left\{\int_{a}^{b}\left|D^{2-\alpha}[G(t, s) q(s)]\right| d s\right\}>1,
$$

where $D^{2-\alpha}[G(t, s) q(s)]$ is the right-sided fractional derivative of $G(t, s) q(s)$ with respect to $s$ with

$$
G(t, s)=\frac{1}{b-a} \begin{cases}(t-a)(b-s), & a \leq t \leq s \leq b \\ (s-a)(b-t), & a \leq s \leq t \leq b .\end{cases}
$$

(b) If problem (58) has a nontrivial solution and $D^{\alpha-2} y(t) \neq 0$ on $(a, b)$, then

$$
\max _{t \in[a, b]}\left\{\int_{a}^{b}\left[D^{2-\alpha}[G(t, s) q(s)]\right]_{+} d s\right\}>1,
$$

where $D^{2-\alpha}[G(t, s) q(s)]_{+}$is the the positive part of $D^{2-\alpha}[G(t, s) q(s)]$.

As a special case we have the following corollary.

Corollary 7. Assume that $D_{b-}^{2-\alpha}[G(t, s) q(s)] \geq 0$ for $t, s \in[a, b]$ so that the problem (58) has a nontrivial solution. Then

$$
\int_{a}^{b} q_{+}(t) d t>\frac{\alpha^{\alpha} \Gamma(\alpha-1)}{(\alpha-1)^{\alpha-1}(b-a)^{\alpha-1}} .
$$

Next we consider the sequential fractional boundary value problem

$$
\left\{\begin{array}{l}
{\left[\left(D_{a+}^{\beta}\left(D^{\alpha} y\right)\right)\right](t)+q(t) y(t)=0, \quad a<t<b, 0<\alpha, \beta \leq 1} \\
\left(D^{\alpha-1} y\right)\left(a^{+}\right)=\left(D^{\alpha-1} y\right)(b)=0
\end{array}\right.
$$

which is equivalent to the integral equation

$$
y(t)=\int_{a}^{b} G(t, s) q(s) D_{a+}^{1-\alpha} y(s) d s,
$$

where

$$
G(t, s)=\frac{1}{\Gamma(\beta+1)} \begin{cases}\frac{(t-a)^{\beta}(b-s)^{\beta}}{(b-a)^{\beta}}, & a \leq t \leq s \leq b, \\ \frac{(t-a)^{\beta}(b-s)^{\beta}}{(b-a)^{\beta}}-(t-s)^{\beta}, & a \leq s \leq t \leq b .\end{cases}
$$

In the following result, we express the fractional Lyapunov-type inequalities for problem (59).

Theorem 13. (a) If problem (59) has a nontrivial solution, then 


$$
\max _{t \in[a, b]}\left\{\int_{a}^{b}\left|D^{1-\alpha}[G(t, s) q(s)]\right| d s\right\}>1
$$

(b) If problem (59) has a nontrivial solution and $\left(D_{a+}^{\alpha-1} y\right)(t) \neq 0$ on $(a, b)$, then

$$
\max _{t \in[a, b]}\left\{\int_{a}^{b}\left[D^{1-\alpha}[G(t, s) q(s)]\right]_{+} d s\right\}>1
$$

As a special case we have the following corollary.

Corollary 8. Assume that $D^{1-\alpha}[G(t, s) q(s)] \geq 0$ for $t, s \in[a, b], 1<\alpha+\beta \leq 2$ and the problem (59) has a nontrivial solution. Then

$$
\int_{a}^{b} q_{+}(t) d t>\frac{(\alpha+2 \beta-1)^{\alpha+2 \beta-1} \Gamma(\alpha) \Gamma(\beta+1)}{(\alpha+\beta-1)^{\alpha+\beta-1} \beta^{\beta}(b-a)^{\alpha+\beta-1}}
$$

For some similar results on fractional boundary value problems of order $\alpha \in$ $(2,3]$, see [28].

In 2017, Jleli et al. [29] obtained Lyapunov-type inequality for higher order fractional boundary value problem

$$
\left\{\begin{array}{l}
D^{\alpha} y(t)+q(t) y(t)=0, \quad a<t<b, \\
y(a)=y^{\prime}(a)=\ldots=y^{(n-2)}(a)=0, y(b)=I^{\alpha}(y h)(b),
\end{array}\right.
$$

where $n \in \mathbb{N}, n-1<\alpha<n, D^{\alpha}$ is the standard Riemann-Liouville fractional derivative of order $\alpha, I^{\alpha}$ denotes the Riemann-Liouville fractional integral of order $\alpha$, and $q, h:[a, b] \rightarrow \mathbb{R}$ are continuous functions.

The function $y$ is a solution of the boundary value problem 60 if and only if $y$ satisfies the integral equation

$$
y(t)=\int_{a}^{b} G(t, s)(q(s)+h(s)) y(s) d s+\frac{1}{\Gamma(\alpha)} \int_{a}^{t}(t-s)^{\alpha-1} h(s) y(s) d s,
$$

where $G(t, s)$ is the Green's function given by 11 such that

$$
0 \leq G(t, s) \leq G\left(s^{*}, s\right)=\frac{(s-a)^{\alpha-1}(b-s)^{\alpha-1}}{\Gamma(\alpha)(b-a)^{\alpha-1}\left[1-\left(\frac{b-s}{b-a}\right)^{\frac{\alpha-1}{\alpha-2}}\right]^{\alpha-2}}, a<s<b,
$$

with

$$
s^{*}=\frac{s-a\left(\frac{b-s}{b-a}\right)^{\frac{\alpha-1}{\alpha-2}}}{1-\left(\frac{b-s}{b-a}\right)^{\frac{\alpha-1}{\alpha-2}}} .
$$

The following result presents the Lyapunov-type inequality for problem 60. 
Theorem 14. Let $n \in \mathbb{N}$ with $n \geq 3$. If $y$ is a nontrivial solution of the fractional boundary value problem (60), then

$$
\int_{a}^{b}\left(|q(s)+h(s)|+\frac{\left(1-z_{\alpha}^{\frac{\alpha-1}{\alpha-2}}\right)^{\alpha-2}}{z_{\alpha}^{\alpha-1}\left(1-z_{\alpha}\right)^{\alpha-1}}|h(s)|\right) d s \geq \frac{\Gamma(\alpha)}{(b-a)^{\alpha-1}} \frac{\left(1-z_{\alpha}^{\frac{\alpha-1}{\alpha-2}}\right)^{\alpha-2}}{z_{\alpha}^{\alpha-1}\left(1-z_{\alpha}\right)^{\alpha-1}},
$$

where $z_{\alpha}$ is the unique zero of the nonlinear algebraic equation

$$
z^{\frac{2 \alpha-3}{\alpha-2}}-2 z+1=0
$$

in the interval $\left(0,\left(\frac{2 \alpha-4}{2 \alpha-3}\right)^{\frac{\alpha-2}{\alpha-1}}\right)$.

Theorem 15. Let $n=2$. If y is a nontrivial solution of the fractional boundary value problem

$$
\left\{\begin{array}{l}
D^{\alpha} y(t)+q(t) y(t)=0, \quad a<t<b \\
y(a)=0, y(b)=I^{\alpha}(h y)(b)
\end{array}\right.
$$

then

$$
\int_{a}^{b}\left(|q(s)+h(s)|+4^{\alpha-1} h(s) \mid\right) d s \geq \Gamma(\alpha)\left(\frac{4}{b-a}\right)^{\alpha-1}
$$

In 2017, Cabrera et al. [30] obtained a Lyapunov-type inequality for a sequential fractional boundary value problem

$$
\left\{\begin{array}{l}
{ }^{C} D^{\alpha} y(t)+q(t) y(t)=0, \quad a<t<b, \quad \alpha \in(n-1, n], n \geq 4, \\
y^{i}(a)=y^{\prime \prime}(b)=0, \quad 0 \leq i \leq n-1, i \neq 2,
\end{array}\right.
$$

where ${ }^{C} D^{\alpha}$ is the Caputo fractional derivative of fractional order $\alpha \geq 0$ and $q$ : $[a, b] \rightarrow \mathbb{R}$ is a continuous function.

The function $y \in C([a, b], \mathbb{R})$ is a solution of the boundary value problem 65 if and only if it satisfies the integral equation

$$
y(t)=\int_{a}^{b} G(t, s) q(s) y(s) d s,
$$

where $G(t, s)$ is the Green's function defined by

$$
G(t, s)=\frac{1}{\Gamma(\alpha)} \begin{cases}\frac{1}{2}(\alpha-1)(\alpha-2)(t-a)^{2}(b-s)^{\alpha-3}-(t-s)^{\alpha-1}, & a \leq s \leq t \leq b, \\ \frac{1}{2}(\alpha-1)(\alpha-2)(t-a)^{2}(b-s)^{\alpha-3}, & a \leq t \leq s \leq b,\end{cases}
$$

such that $G(t, s) \geq 0$ for $t, s \in[a, b],|G(t, s)| \leq G(b, s)$ for $t, s \in[a, b]$ and

$$
|G(t, s)| \leq G(b, s)=\frac{1}{2}(\alpha-1)(\alpha-2)(b-a)^{2}(b-s)^{\alpha-3}-(b-s)^{\alpha-1}, \quad(t, s) \in[a, b] \times[a, b] .
$$


Their result is as follows.

Theorem 16. If there exists a nontrivial continuous solution of the fractional boundary value problem (65), then

$$
\int_{a}^{b}\left[\frac{1}{2}(\alpha-1)(\alpha-2)(b-a)^{2}(b-s)^{\alpha-3}-(b-s)^{\alpha-1}\right]|q(s)| d s \geq \Gamma(\alpha) .
$$

In 2017, Wang et al. [31] obtained a Lyapunov-type inequality for the higher order fractional boundary value problem

$$
\left\{\begin{array}{l}
D^{\alpha} y(t)+q(t) y(t)=0, \quad a<t<b \\
y(a)=y^{\prime}(a)=\ldots=y^{(n-2)}(a)=0, y^{(n-2)}(b)=0
\end{array}\right.
$$

where $n \in \mathbb{N}, 2<n-1<\alpha \leq n, D^{\alpha}$ is the standard Riemann-Liouville fractional derivative of order $\alpha$, and $q:[a, b] \rightarrow \mathbb{R}$ is a continuous function.

The function $y$ is a solution of the boundary value problem 69 if and only if $y$ satisfies the integral equation

$$
y(t)=\int_{a}^{b} G(t, s) q(s) y(s) d s
$$

where $G(t, s)$ is the Green's function given by

$$
G(t, s)=\frac{1}{\Gamma(\alpha)} \begin{cases}\frac{(t-a)^{\alpha-1}(b-s)^{\alpha-n+1}}{(b-a)^{\alpha-n+1}}, & a \leq t \leq s \leq b, \\ \frac{(t-a)^{\alpha-1}(b-s)^{\alpha-n+1}}{(b-a)^{\alpha-n+1}}-(t-s)^{\alpha-1}, & a \leq s \leq t \leq b,\end{cases}
$$

such that

$0 \leq G(t, s) \leq G(b, s)=\frac{(b-s)^{\alpha-n+1}(s-a)}{\Gamma(\alpha)} \sum_{i=1}^{n-2}(-1)^{i-1} C_{n-2}^{i}(b-a)^{n-2-i}(s-a)^{i-1}$,

$(t, s) \in[a, b] \times[a, b]$ and $C_{n-2}^{i}$ is the binomial coefficient.

Their Lyapunov-type inequality for the problem 69] is given in the following theorem.

Theorem 17. If there exists a nontrivial continuous solutions y of the fractional boundary value problem (69), and q is a real continuous function, then

$$
\int_{a}^{b}(b-s)^{\alpha-n+1}(s-a) \sum_{i=1}^{n-2}(-1)^{i-1} C_{n-2}^{i}(b-a)^{n-2-i}(s-a)^{i-1}|q(s)| d s \geq \Gamma(\alpha) .
$$

Corollary 9. If the fractional boundary value problem (69) has a nontrivial continuous solution, then 


$$
\int_{a}^{b}|q(s)| d s \geq \frac{\Gamma(\alpha)(\alpha-n+2)^{\alpha-n+2}}{(n-2)(\alpha-n+1)^{\alpha-n+1}(b-a)^{\alpha-1}} .
$$

The following result shows the application of the above Lyapunov-type inequality to eigenvalue problems.

Corollary 10. If $\lambda$ is an eigenvalue to the fractional boundary value problem

$$
\left\{\begin{array}{l}
D^{\alpha} y(t)+\lambda y(t)=0, \quad a<t<b, \\
y(a)=y^{\prime}(a)=\ldots=y^{(n-2)}(a)=0, y^{(n-2)}(b)=0,
\end{array}\right.
$$

then

$$
|\lambda| \geq \frac{\Gamma(\alpha)(\alpha-n+3)(\alpha-n+2)}{n-2} .
$$

In 2017, Fereira [32] obtained a Lyapunov-type inequality for so-called antiperiodic boundary value problem:

$$
\left\{\begin{array}{l}
{ }^{C} D^{\alpha} y(t)+q(t) y(t)=0, \quad a<t<b \\
y(a)+y(b)=0=y^{\prime}(a)+y^{\prime}(b)
\end{array}\right.
$$

where ${ }^{C} D^{\alpha}$ is the Caputo fractional derivative of order $1<\alpha \leq 2$ and $q:[a, b] \rightarrow \mathbb{R}$ is a continuous function.

Then $y \in C([a, b], \mathbb{R})$ is a solution of the boundary value problem $[70$ if and only if it satisfies the integral equation

$$
y(t)=\int_{a}^{b}(b-s)^{\alpha-2} H(t, s) q(s) y(s) d s,
$$

where $H(t, s)$ is defined by

$$
H(t, s)=\frac{1}{\Gamma(\alpha)} \begin{cases}\left(-\frac{b-a}{4}+\frac{t-a}{2}\right)(\alpha-1)+\frac{b-s}{2}, & a \leq t \leq s \leq b, \\ \left(-\frac{b-a}{4}+\frac{t-a}{2}\right)(\alpha-1)+\frac{b-s}{2}-\frac{(t-s)^{\alpha-1}}{(b-a)^{\alpha-2}}, & a \leq s \leq t \leq b .\end{cases}
$$

Here the function $H$ satisfies the following property:

$$
|H(t, s)| \leq \frac{(b-a)(3-\alpha)}{4}, \quad(t, s) \in[a, b] \times[a, b] .
$$

The Lyapunov-type inequality for the problem (70) is given in the following result.

Theorem 18. If (70) admits a nontrivial continuous solution, then

$$
\int_{a}^{b}(b-s)^{\alpha-2}|q(s)| d s \geq \frac{4}{(b-a)(3-\alpha)} .
$$


Inequality (73) is useful in finding a bound for the possible eigenvalues of the fractional boundary value problem:

$$
\left\{\begin{array}{l}
D^{\alpha} y(t)+\lambda y(t)=0, a<t<b \\
y(a)+y(b)=0=y^{\prime}(a)+y^{\prime}(b)
\end{array}\right.
$$

that is, an eigenvalue $\lambda \in \mathbb{R}$ satisfies the inequality

$$
|\lambda|>\frac{4(\alpha-1)}{(b-a)^{\alpha}(3-\alpha)} .
$$

In 2017, Agarwal and Zbekler [33] obtained a Lyapunov-type inequality for the following fractional boundary value problem with Dirichlet boundary conditions

$$
\left\{\begin{array}{l}
\left(D^{\alpha} y\right)(t)+p(t)|y(t)|^{\mu-1} y(t)+q(t)|y(t)|^{\gamma-1} y(t)=f(t), \quad a<t<b, \\
y(a)=0, \quad y(b)=0
\end{array}\right.
$$

where $D^{\alpha}$ is the Riemann-Liouville fractional derivative, $p, q, f \in C\left[t_{0}, \infty\right)$ and $0<$ $\gamma<1<\mu<2$. No sign restrictions are imposed on the potentials $p$ and $q$, and the forcing term $f$.

The problem (74) is equivalent to the following integral equation

$$
y(t)=\int_{a}^{b} G(t, s)\left[p(s) y^{\mu}(s)+q(s) y^{\gamma}(s)-f(s)\right] d s,
$$

where $G(t, s)$ is the Green's function defined by (11).

Their Lyapunov-type inequality for the problem (74) is as follows.

Theorem 19. Let $y$ be a nontrivial solution of the problem (74). If $y(t) \neq 0$ in $(a, b)$, then the inequality

$$
\left(\int_{a}^{b}\left[p^{+}(t)+q^{+}(t)\right] d t\right)\left(\int_{a}^{b}\left[\mu_{0} p^{+}(t)+\gamma_{0} q^{+}(t)+|f(t)|\right] d t\right)>\frac{4^{2 \alpha-3} \Gamma^{2}(\alpha)}{(b-a)^{2 \alpha-2}}
$$

holds, where $u^{+}=\max \{u, 0\}, u=p, q$ and

$$
\mu_{0}=(2-\mu) \mu^{\mu /(2-\mu)} 2^{2 /(\mu-2)}>0, \quad \gamma_{0}=(2-\gamma) \gamma^{\gamma /(2-\gamma)} 2^{2 /(\gamma-2)}>0 .
$$

In 2017, Zhang and Zheng [34] considered the Riemann-Liouville fractional differential equations with mixed nonlinearities of order $\alpha \in(n-1, n]$ for $n \geq 3$

$$
\left(D^{\alpha} y\right)(t)+p(t)|y(t)|^{\mu-1} y(t)+q(t)|y(t)|^{\gamma-1} y(t)=f(t),
$$

where $p, q, f \in C\left(\left[t_{0}, \infty\right), \mathbb{R}\right)$ and the constants satisfy $0<\gamma<1<\mu<n(n \geq 3)$. Equation (76) subjects to the following two kinds boundary conditions, respectively:

$$
y(a)=y^{\prime}(a)=y^{\prime \prime}(a)=\ldots=y^{(n-2)}(a)=y(b)=0,
$$


and

$$
y(a)=y^{\prime}(a)=y^{\prime \prime}(a)=\ldots=y^{(n-2)}(a)=y^{\prime}(b)=0,
$$

where $a$ and $b$ are two consecutive zeros of the function $y$.

Obviously, it is easy to see that equation (76) has two special forms; one is the forced sub-linear $(p(t)=0)$ fractional equation

$$
\left(D^{\alpha} y\right)(t)+q(t)|y(t)|^{\gamma-1} y(t)=f(t), 0<\gamma<1,
$$

and the other is the forced super-linear $(q(t)=0)$ fractional equation

$$
\left(D^{\alpha} y\right)(t)+q(t)|y(t)|^{\gamma-1} y(t)=f(t), 1<\mu<n .
$$

Their Lyapunov-type inequalities for the problems (76)-(77) and (76)-(78) are respectively the following:

Theorem 20. Let y be a positive solution of the boundary value problem (76)-(77) in $(a, b)$. Then

$$
\begin{aligned}
& \left(\int_{a}^{b}[(b-s)(s-a)]^{\alpha-1}\left[1-\left(\frac{b-s}{b-a}\right)^{\frac{\alpha-1}{\alpha-2}}\right]^{2-\alpha}\left[\mu_{0} p^{+}(s)+\gamma_{0} q^{+}(s)+f^{-}(s)\right] d s\right) \\
& \times\left(\int_{a}^{b}[(b-s)(s-a)]^{\alpha-1}\left[1-\left(\frac{b-s}{b-a}\right)^{\frac{\alpha-1}{\alpha-2}}\right]^{2-\alpha}\left[p^{+}(s)+q^{+}(s)\right] d s\right) \\
& >\left[\Gamma(\alpha)(b-a)^{\alpha-1}\right]^{\frac{n}{n-1}}(n-1) n^{1-n} \\
& \text { where } \mu_{0}=(n-\mu) \mu^{\frac{\mu}{n-\mu}} n^{\frac{n}{n-\mu}} \text { and } \gamma_{0}=(n-\gamma) \gamma^{\frac{\gamma}{n-\gamma}} n^{\frac{n}{\gamma-n}} .
\end{aligned}
$$

Theorem 21. Let y be a positive solution of the boundary value problem (76)-(78) in $(a, b)$. Then

$$
\begin{aligned}
& \left(\int_{a}^{b}\left[(b-s)^{\alpha-2}(s-a)\right]\left[\mu_{0} p^{+}(s)+\gamma_{0} q^{+}(s)+f^{-}(s)\right] d s\right) \\
& \left.\times\left([b-s)^{\alpha-2}(s-a)\right]\left[p^{+}(s)+q^{+}(s)\right] d s\right)^{\frac{1}{\mu-1}} \\
& >\Gamma(\alpha)^{\frac{n}{n-1}}(n-1) n^{\frac{n}{1-n}}
\end{aligned}
$$

where $\mu_{0}$ and $\gamma_{0}$ are the same as in Theorem 21$]$

In 2017, Chidouh and Torres [35] extended the linear term $q(t) y(t)$ to a nonlinear term of the form $q(t) f(y(t))$ and obtained a generalized Lyapunov's inequality for the fractional boundary value problem

$$
\left\{\begin{array}{l}
\left(D^{\alpha} y\right)(t)+q(t) f(y(t))=0, \quad a<t<b, \quad 1<\alpha \leq 2, \\
y(a)=y(b)=0,
\end{array}\right.
$$


where $D^{\alpha}$ is the Riemann-Liouville fractional derivative of order $\alpha, f: \mathbb{R}^{+} \rightarrow \mathbb{R}^{+}$ is continuous and $q:[a, b] \rightarrow \mathbb{R}^{+}$is a Lebesgue integrable function.

An integral equation equivalent to the Problem (81) is

$$
y(t)=\int_{a}^{b} G(t, s) q(s) f(y(s)) d s,
$$

where $G(t, s)$ is the Green's function defined by (11) and satisfies the following properties:

(i) $G(t, s) \geq 0$, for all $(t, s) \in[a, b] \times[a, b]$;

(ii) $\max _{t \in[a, b]} G(t, s)=G(s, s), s \in[a, b]$;

(iii) $G(s, s)$ has a unique maximum given by $\max _{s \in[a, b]} G(s, s)=\frac{(b-a)^{\alpha-1}}{4^{\alpha-1} \Gamma(\alpha)}$.

The Lyapunov-type inequality for the problem $[81$ is as follows.

Theorem 22. Let $q$ be a real nontrivial Lebesgue integrable function. Assume that $f \in C\left(\mathbb{R}^{+}, \mathbb{R}^{+}\right)$is a concave and nondecreasing function. If the fractional boundary value problem (81) has a nontrivial solution $y$, then

$$
\int_{a}^{b} q(s) d s>\frac{4^{\alpha-1} \Gamma(\alpha) \eta}{(b-a)^{\alpha-1} f(\eta)} .
$$

where $\eta=\max _{t \in[a, b]} y(t)$.

In 2016, Ma [36] obtained a generalized form of Lyapunov's inequality for the fractional boundary value problem

$$
\left\{\begin{array}{l}
\left(D^{\alpha} y\right)(t)+q(t) f(y(t))=0, \quad a<t<b, \quad 1<\alpha \leq 2, \\
y(a)=y(b)=y^{\prime \prime}(a)=0,
\end{array}\right.
$$

where $D^{\alpha}$ is the Riemann-Liouville fractional derivative of order $\alpha, q:[a, b] \rightarrow \mathbb{R}^{+}$ is a Lebesgue integrable function and $f: \mathbb{R}^{+} \rightarrow \mathbb{R}^{+}$is continuous.

The function $y \in C([a, b], \mathbb{R})$ is a solution to the problem 83 if and only if $y$ satisfies the integral equation

$$
y(t)=\int_{a}^{b} G(t, s) q(s) f(y(s)) d s,
$$

where $G(t, s)$ is the Green's function defined by

$$
G(t, s)=\frac{1}{\Gamma(\alpha)} \begin{cases}\frac{(b-s)^{\alpha-1}(t-a)}{(b-a)}, & a \leq t \leq s \leq b, \\ \frac{(b-s)^{\alpha-1}(t-a)}{(b-a)}-(t-s)^{\alpha-1}, & a \leq s \leq t \leq b .\end{cases}
$$

The above Green's function satisfies the following properties: 
(i) $G(t, s) \geq 0$, for all $(t, s) \in[a, b] \times[a, b]$;

(ii) For any $s \in[a, b]$,

$$
\max _{t \in[a, b]} G(t, s)=G\left(t_{0}, s\right)=\frac{(s-a)(b-s)^{\alpha-1}}{\Gamma(\alpha)(b-a)}+\frac{(\alpha-2)(b-s)^{\frac{(\alpha-1)^{2}}{\alpha-2}}}{(\alpha-1)^{\frac{\alpha-1}{\alpha-2}}(b-a)^{\frac{\alpha-1}{\alpha-2}} \Gamma(\alpha)},
$$

where $t_{0}=s+\left(\frac{(b-s)^{\alpha-1}}{(b-a)(\alpha-1)}\right)^{\frac{1}{\alpha-2}} \in[s, b]$

(iii) $\max _{s \in[a, b]} G\left(t_{0}, s\right) \leq \frac{(b-a)^{\alpha-1}}{\Gamma(\alpha)}$;

(iv) $G(t, s) \geq \frac{(t-a)(b-t)}{(b-a)^{2}} G\left(t_{0}, s\right)$ for all $a \leq t, s \leq b$.

The following Lyapunov-type inequalities are given in [36].

Theorem 23. Assume that $f$ is bounded by two lines, that is, there exist two positive constants $M$ and $N$ such that $N y \leq f(y) \leq M y$ for any $y \in \mathbb{R}^{+}$. If (83) has a solution in $E^{+}=\{y \in C[a, b], y(t) \geq 0$, for any $t \in[a, b]$ and $\|y\| \neq 0\}$, then the following Lyapunov-type inequalities hold:

(i) $\int_{a}^{b} q(s) d s>\frac{\Gamma(\alpha)}{M(b-a)^{\alpha-1}}$;

(ii) $\int_{a}^{b}(s-a)^{2}(b-s)^{\alpha} q(s) d s \leq \frac{4 \Gamma(\alpha)(b-a)^{3}}{N}$;

(iii) $\int_{a}^{b}(s-a)(b-s)^{\frac{\alpha^{2}-\alpha-1}{\alpha-2}} q(s) d s \leq \frac{4 \Gamma(\alpha)(b-a)^{\frac{3 \alpha-2}{\alpha-2}}(\alpha-1)^{\frac{\alpha-1}{\alpha-2}}}{(\alpha-2) N}$.

The applications of the above inequalities are given in the following corollaries.

Corollary 11. For any $\lambda \in[0, \Gamma(v)] \cup\left(\frac{4 \Gamma(v)}{B(3, v+1)},+\infty\right)$, where $B(x, y)=\int_{0}^{1} s^{x-1}(1-$ $s)^{y-1} d s, x>0, y>0$, the eigenvalue for the problem

$$
\left\{\begin{array}{l}
\left(D^{v} y\right)(t)+\lambda y(t)=0, \quad 0<t<1,2<v \leq 3 \\
y(0)=y(1)=y^{\prime \prime}(0)=0
\end{array}\right.
$$

has no corresponding eigenfunction $y \in E^{+}$.

In the next Corollary we obtain an interval in which the Mittag-Leffler function $E_{v, 2}(z)$ with $\beta=2,2<v \leq 3$ has no real zeros.

Corollary 12. Let $2<v \leq 3$. Then the Mittag-Leffler function $E_{v, 2}(z)=\sum_{k=0}^{\infty} \frac{z^{k}}{\Gamma(k v+2)}$ has no real zeros for $z \in\left(-\infty,-\frac{4 \Gamma(v)}{B(3, v+1},\right) \cup[-\Gamma(v),+\infty)$.

In 2017, Ru et al. [37] obtained the Lyapunov-type inequality for the following fractional Sturm-Liouville boundary value problem 


$$
\left\{\begin{array}{l}
D_{0^{+}}^{\alpha}\left(p(t) y^{\prime}(t)\right)+q(t) y(t)=0, \quad 0<t<1,1<\alpha \leq 2 \\
a y(0)-b p(0) y^{\prime}(0)=0, \quad c y(1)+d p(1) y^{\prime}(1)=0
\end{array}\right.
$$

where $a, b, c, d>0 D^{\alpha}$ is the standard Riemann-Liouville fractional derivative of order $\alpha, p:[0,1] \rightarrow(0, \infty)$ and $q:[0,1] \rightarrow \mathbb{R}$ is a nontrivial Lebesgue integrable function.

The solution of the boundary value problem $(86)$ in terms of the integral equation is

$$
y(t)=\int_{a}^{b} G(t, s) q(s) y(s) d s
$$

where $G(t, s)$ is the Green's function given by

$$
\begin{gathered}
G(t, s)=\frac{1}{\rho \Gamma(\alpha)} \begin{cases}{\left[b+a \int_{0}^{t} \frac{d \tau}{p(\tau)}\right]\left[d(1-s)^{\alpha-1}+c \int_{s}^{1} \frac{(\tau-s)^{\alpha-1} d \tau}{p(\tau)}\right],} & 0 \leq t \leq s \leq 1, \\
{\left[b+a \int_{0}^{t} \frac{d \tau}{p(\tau)}\right]\left[d(1-s)^{\alpha-1}+c \int_{s}^{1} \frac{(\tau-s)^{\alpha-1} d \tau}{p(\tau)}\right]-H(t, s),} & 0 \leq s \leq t \leq 1,\end{cases} \\
\rho=b c+a c \int_{0}^{1} \frac{1}{p(\tau)} d \tau+a d, H(t, s)=a\left[d+c \int_{t}^{1} \frac{d \tau}{p(\tau)}\right] \int_{0}^{t} \frac{(\tau-s)^{\alpha-1}}{p(\tau)} d \tau .
\end{gathered}
$$

Further, the above Green's function $G(t, s)$ satisfies the following properties:

(i) $G(t, s) \geq 0$ for $0 \leq t, s \leq 1$;

(ii) The maximum value of $G(t, s)$ is

$$
\bar{G}=\max _{0 \leq t, s \leq 1} G(t, s)=\max \left\{\max _{s \in[0,1]} G(s, s), \max _{s \in[0,1]} G\left(t_{0}(s), s\right)\right\}
$$

where

$$
t_{0}(s)=s+\left[\frac{a d(1-s)^{\alpha-1}+a c \int_{s}^{1} \frac{(\tau-s)^{\alpha-1}}{p(\tau)} d \tau}{\rho}\right]^{\frac{1}{\alpha-1}} .
$$

They obtained the following Lyapunov-type inequality for the problem 86 .

Theorem 24. For any nontrivial solutions y of the fractional boundary value problem (86), the following Lyapunov-type inequality holds:

$$
\int_{0}^{1}|q(s)| d s>\frac{1}{\bar{G}}
$$

where $\bar{G}$ is defined by (87).

In [37], the authors also considered the generalized fractional Sturm-Liouville boundary value problem:

$$
\left\{\begin{array}{l}
D_{0^{+}}^{\alpha}\left(p(t) y^{\prime}(t)\right)+q(t) f(y(t))=0, \quad 0<t<1,1<\alpha \leq 2 \\
a y(0)-b p(0) y^{\prime}(0)=0, \quad c y(1)+d p(1) y^{\prime}(1)=0
\end{array}\right.
$$


where $f: \mathbb{R} \rightarrow \mathbb{R}$ is a continuous function, and obtained the Lyapunov-type inequality for this problem as follows.

Theorem 25. Let $f$ is a positive function on $\mathbb{R}$. For any nontrivial solutions $y$ of the fractional boundary value problem (88), the following Lyapunov-type inequality will be satisfied

$$
\int_{0}^{1}|q(s)| d s>\frac{y^{*}}{\bar{G} \max _{y \in\left[y_{*} \cdot y^{*}\right]} f(y)},
$$

where $\bar{G}$ is defined by (87) and $y_{*}=\min _{t \in[0,1]} y(t), y^{*}=\max _{t \in[0,1]} y(t)$.

\section{Lyapunov inequalities for nonlocal boundary value problems}

In 2017, Cabrera et al. [38] obtained Lyapunov-type inequalities for a nonlocal fractional boundary value problem

$$
\left\{\begin{array}{l}
{ }^{C} D^{\alpha} y(t)+q(t) y(t)=0, \quad a<t<b, \\
y^{\prime}(a)=0, \quad \beta^{C} D^{\alpha-1} y(b)+y(\eta)=0,
\end{array}\right.
$$

where ${ }^{C} D^{\alpha}$ is the Caputo fractional derivative of fractional order $1<\alpha \leq 2, \beta>$ $0, a<\eta<b, \beta>\frac{(\beta-\eta)^{\alpha-1}}{\Gamma(\alpha)}$ and $q:[a, b] \rightarrow \mathbb{R}$ is a continuous function.

Note that problem (89) is the fractional analogue of the classical nonlocal boundary value problem

$$
\left\{\begin{array}{l}
y^{\prime \prime}(t)+q(t) y(t)=0, \quad 0<t<1, \\
y^{\prime}(0)=0, \quad \beta y^{\prime}(1)+y(\eta)=0,0<\eta<1,
\end{array}\right.
$$

which represents a thermostat model insulated at $t=0$ with a controller dissipating heat at $t=1$ depending on the temperature detected by a sensor at $t=\eta[39]$.

The function $y \in C([a, b], \mathbb{R})$ is a solution of the boundary value problem 896 if and only if $y$ satisfies the integral equation

$$
y(t)=\int_{a}^{b} G(t, s) q(s) y(s) d s,
$$

where $G(t, s)$ is the Green's function defined by

$$
G(t, s)=\beta+H_{\eta}(s)-H_{t}(s),
$$

for $r \in[a, b], H_{r}:[a, b] \rightarrow \mathbb{R}$ is 


$$
H_{r}(s)= \begin{cases}\frac{(r-s)^{\alpha-1}}{\Gamma(\alpha)}, & a \leq s \leq r \leq b \\ 0, & a \leq r \leq s \leq b\end{cases}
$$

The Green's function defined in (92) satisfies the relation

$$
|G(t, s)| \leq \beta+\frac{(\eta-a)^{\alpha-1}}{\Gamma(\alpha)}, \quad(t, s) \in[a, b] \times[a, b] .
$$

The Lyapunov-type inequality derived for the problem 89 is given in the following result.

Theorem 26. If there exists a nontrivial continuous solution of the fractional boundary value problem (89), then

$$
\int_{a}^{b}|q(s)| d s>\frac{\Gamma(\alpha)}{\beta \Gamma(\alpha)+(\eta-\alpha)^{\alpha-1}} .
$$

In 2017, Cabrera et al. [40] obtained a Lyapunov-type inequality for the following nonlocal fractional boundary value problem

$$
\left\{\begin{array}{l}
\left(D^{\alpha} y\right)(t)+q(t) y(t)=0, \quad a<t<b, \quad 2<\alpha \leq 3 \\
y(a)=y^{\prime}(a)=0, \quad y^{\prime}(b)=\beta y(\xi)
\end{array}\right.
$$

where $D^{\alpha}$ is the Riemann-Liouville fractional derivative of order $\alpha, a<\xi<b$, $0 \leq \beta(\xi-a)^{\alpha-1}<(\alpha-1)(b-a)^{\alpha-2}$, and $q:[a, b] \rightarrow \mathbb{R}$ is a continuous function.

The unique solution of the nonlocal boundary value problem 94 is given by

$$
y(t)=\int_{a}^{b} G(t, s) q(s) y(s) d s+\frac{\beta(t-a)^{\alpha-1}}{(\alpha-1)(b-a)^{\alpha-2}-\beta(\xi-a)^{\alpha-1}} \int_{a}^{b} G(\xi, s) q(s) y(s) d s,
$$

where $G(t, s)$ is the Green's function defined by

$$
G(t, s)=\frac{1}{\Gamma(\alpha)} \begin{cases}\frac{(b-s)^{\alpha-2}(t-a)^{\alpha-1}}{(b-a)^{\alpha-2}}, & a \leq t \leq s \leq b, \\ \frac{(b-s)^{\alpha-2}(t-a)^{\alpha-1}}{(b-a)^{\alpha-2}}-(t-s)^{\alpha-1}, & a \leq s \leq t \leq b .\end{cases}
$$

The Green's function defined in (95) satisfies the following properties:

(i) $G(t, s) \geq 0$, for all $(t, s) \in[a, b] \times[a, b]$;

(ii) $G(t, s)$ is non-decreasing with respect to the first variable;

(iii) $0 \leq G(a, s) \leq G(t, s) \leq G(b, s),(t, s) \in[a, b] \times[a, b]$.

Their Lyapunov-type inequality for the problem 94 is expressed as follows.

Theorem 27. If the problem (94) has a nontrivial solution, then 


$$
\int_{a}^{b}|q(s)| d s \geq \frac{\Gamma(\alpha)(\alpha-1)^{\alpha-1}}{(b-a)^{\alpha-1}(\alpha-2)^{\alpha-2}}\left(1+\frac{\beta(b-a)^{\alpha-1}}{(\alpha-1)(b-a)^{\alpha-2}-\beta(\xi-a)^{\alpha-1}}\right)^{-1} .
$$

As an application of Theorem 27, we consider the following eigenvalue problem

$$
\left\{\begin{array}{l}
D^{\alpha} y(t)+\lambda y(t)=0, \quad a<t<b, 2<\alpha \leq 3 \\
y(a)=y^{\prime}(a)=0, y^{\prime}(b)=\beta y(\xi)
\end{array}\right.
$$

where $a<\xi<b$ and $0 \leq \beta(\xi-a)^{\alpha-1}<(\alpha-1)(b-a)^{\alpha-2}$. If $\lambda$ is an eigenvalue of problem 97 , then

$$
|\lambda| \geq \frac{\alpha(\alpha-1) \Gamma(\alpha)}{(b-a)^{\alpha}}\left(1+\frac{\beta(b-a)^{\alpha-1}}{(\alpha-1)(b-a)^{\alpha-2}-\beta(\xi-a)^{\alpha-1}}\right)^{-1} .
$$

This is an immediate consequence of Theorem 27

Very recently, Y. Wang and Q. Wang [41] obtained Lyapunov-type inequalities for the fractional differential equations with multi-point boundary conditions

$$
\left\{\begin{array}{l}
D^{\alpha} y(t)+q(t) y(t)=0, \quad a<t<b, 2<\alpha \leq 3 \\
y(a)=y^{\prime}(a)=0, \quad\left(D^{\beta+1} y\right)(b)=\sum_{i=1}^{m-2} b_{i}\left(D^{\beta} y\right)\left(\xi_{i}\right)
\end{array}\right.
$$

where $D^{\alpha}$ denotes the standard Riemann-Liouville fractional derivative of order $\alpha$, $\alpha>\beta+2,0<\beta<1, a<\xi_{1}<\xi_{2}<\ldots<\xi_{m-2}<b, b_{i} \geq 0(i=1,2, \ldots, m-2), 0 \leq$ $\sum_{i=1}^{m-2} b_{i}\left(\xi_{i}-a\right)^{\alpha-\beta-1}<(\alpha-\beta-1)(b-a)^{\alpha-\beta-2}$ and $q:[a, b] \rightarrow \mathbb{R}$ is a continuous function. is

The solution of the boundary value problem 98 in terms of the integral equation

$$
y(t)=\int_{a}^{b} G(t, s) q(s) y(s) d s+T(t) \int_{a}^{b}\left(\sum_{i=1}^{m-2} b_{i} H(\xi, s) q(s) y(s)\right) d s,
$$

where $G(t, s), H(t, s)$ and $T(t)$ defined by

$$
\begin{gathered}
G(t, s)=\frac{1}{\Gamma(\alpha)} \begin{cases}\frac{(t-a)^{\alpha-1}(b-s)^{\alpha-\beta-2}}{(b-a)^{\alpha-\beta-2}}-(t-s)^{\alpha-1}, & a \leq s \leq t \leq b, \\
\frac{(t-a)^{\alpha-1}(b-s)^{\alpha-\beta-2}}{(b-a)^{\alpha-\beta-2}}, & a \leq t \leq s \leq b .\end{cases} \\
H(t, s)=\frac{1}{\Gamma(\alpha)} \begin{cases}\frac{(t-a)^{\alpha-\beta-1}(b-s)^{\alpha-\beta-2}}{(b-a)^{\alpha-\beta-2}}-(t-s)^{\alpha-\beta-1}, & a \leq s \leq t \leq b, \\
\frac{(t-a)^{\alpha-\beta-1}(b-s)^{\alpha-\beta-2}}{(b-a)^{\alpha-\beta-2}}, & a \leq t \leq s \leq b .\end{cases}
\end{gathered}
$$




$$
T(t)=\frac{(t-a)^{\alpha-1}}{(\alpha-\beta-1)(b-a)^{\alpha-\beta-2}-\sum_{i=1}^{m-2} b_{i}\left(\xi_{i}-a\right)^{\alpha-\beta-1}}, \quad t \geq a .
$$

Further, the above functions $G(t, s)$ and $H(t, s)$ satisfies the following properties:

(i) $G(t, s) \geq 0$ for $a \leq t, s \leq b$;

(ii) $G(t, s)$ is non-decreasing with respect to the first variable;

(iii) $0 \leq G(a, s) \leq G(t, s) \leq G(b, s)=\frac{1}{\Gamma(\alpha)}(b-s)^{\alpha-\beta-2}\left[(b-a)^{\beta+1}-(b-s)^{\beta+1}\right]$, $(t, s) \in[a, b] \times[a, b]$

(iv) for any $s \in[a, b]$,

$$
\max _{s \in[a, b]} G(b, s)=\frac{\beta+1}{\alpha-1}\left(\frac{\alpha-\beta-2}{\alpha-1}\right)^{(\alpha-\beta-2) /(\beta+1)} \frac{\left(b-a^{\alpha-1}\right.}{\Gamma(\alpha)}
$$

(v) $H(t, s) \geq 0$ for $a \leq t, s \leq b$;

(vi) $H(t, s)$ is non-decreasing with respect to the first variable;

(vii) $\quad 0 \leq H(a, s) \leq H(t, s) \leq H(b, s)=\frac{1}{\Gamma(\alpha)}(b-s)^{\alpha-\beta-2}(s-a),(t, s) \in[a, b] \times$ $[a, b]$

$$
\max _{s \in[a, b]} H(b, s)=H\left(b, s^{*}\right)=\frac{(\alpha-\beta-2)^{\alpha-\beta-2}}{\Gamma(\alpha)}\left(\frac{b-a}{\alpha-\beta-1}\right)^{\alpha-\beta-1},
$$

where $s^{*}=\frac{\alpha-\beta-2}{\alpha-\beta-1} a+\frac{1}{\alpha-\beta-1} b$.

They obtained the following Lyapunov-type inequalities.

Theorem 28. If the fractional boundary value problem

$$
\left\{\begin{array}{l}
D^{\alpha} y(t)+q(t) y(t)=0, \quad a<t<b, 2<\alpha \leq 3 \\
y(a)=y^{\prime}(a)=0, \quad\left(D^{\beta+1} y\right)(b)=\sum_{i=1}^{m-2} b_{i}\left(D^{\beta} y\right)\left(\xi_{i}\right),
\end{array}\right.
$$

has a nontrivial solution, where $q$ is a real and continuous function, then

$\int_{a}^{b}(b-s)^{\alpha-\beta-2}\left[(b-a)^{\beta+1}-(b-s)^{\beta+1}+\sum_{i=1}^{m-2} b_{i} T(b)(s-a)\right]|q(s)| d s \geq \Gamma(\alpha)$.

Note that 


$$
\begin{aligned}
& \Gamma(\alpha)\left[G(b, s)+\sum_{i=1}^{m-2} b_{i} T(b) H(b, s)\right] \\
\leq & \Gamma(\alpha)\left[\max _{s \in[a, b]} G(b, s)+\sum_{i=1}^{m-2} b_{i} T(b) \max _{s \in[a, b]} H(b, s)\right] \\
= & \frac{\beta+1}{\alpha-1}\left(\frac{\alpha-\beta-2}{\alpha-1}\right)^{(\alpha-\beta-2) /(\beta+1)}(b-a)^{\alpha-1} \\
& +\sum_{i=1}^{m-2} b_{i} T(b)(\alpha-\beta-2)^{\alpha-\beta-2}\left(\frac{b-a}{\alpha-\beta-1}\right)^{\alpha-\beta-1} .
\end{aligned}
$$

Thus we have

Corollary 13. If the fractional boundary value problem

$$
\left\{\begin{array}{l}
D^{\alpha} y(t)+q(t) y(t)=0, \quad a<t<b, 2<\alpha \leq 3 \\
y(a)=y^{\prime}(a)=0, \quad\left(D^{\beta+1} y\right)(b)=\sum_{i=1}^{m-2} b_{i}\left(D^{\beta} y\right)\left(\xi_{i}\right),
\end{array}\right.
$$

has a nontrivial solution, where $q$ is a real and continuous function, then

$$
\int_{a}^{b}|q(s)| d s \geq \frac{\Gamma(\alpha)}{\frac{\beta+1}{\alpha-1}\left(\frac{\alpha-\beta-2}{\alpha-1}\right)^{\frac{\alpha-\beta-2}{\beta+1}}(b-a)^{\alpha-1}+\sum_{i=1}^{m-2} b_{i} T(b)(\alpha-\beta-2)^{\alpha-\beta-2}\left(\frac{b-a}{\alpha-\beta-1}\right)^{\alpha-\beta-1}} .
$$

If $\beta=0$ in Theorem 28 we obtain

Corollary 14. If the fractional boundary value problem

$$
\left\{\begin{array}{l}
D^{\alpha} y(t)+q(t) y(t)=0, \quad a<t<b, 2<\alpha \leq 3, \\
y(a)=y^{\prime}(a)=0, \quad y^{\prime}(b)=\sum_{i=1}^{m-2} b_{i} y\left(\xi_{i}\right)
\end{array}\right.
$$

has a nontrivial solution, where $q$ is a real and continuous function, then

$$
\begin{aligned}
& \int_{a}^{b}(b-s)^{\alpha-2}(s-a)|q(s)| d s \\
\geq & \frac{\Gamma(\alpha)}{1+\sum_{i=1}^{m-2} b_{i} T(b)} \\
= & \frac{(\alpha-\beta-1)(b-a)^{\alpha-\beta-2}+\sum_{i=1}^{m-2} b_{i}\left(\xi_{i}-a\right)^{\alpha-\beta-1}}{(\alpha-\beta-1)(b-a)^{\alpha-\beta-2}-\sum_{i=1}^{m-2} b_{i}\left(\xi_{i}-a\right)^{\alpha-\beta-1}+\sum_{i=1}^{m-2} b_{i}(b-a)^{\alpha-1}} \Gamma(\alpha) .
\end{aligned}
$$

If $\beta=0$ in Corollary 13 we have

Corollary 15. If the fractional boundary value problem 


$$
\left\{\begin{array}{l}
D^{\alpha} y(t)+q(t) y(t)=0, \quad a<t<b, 2<\alpha \leq 3, \\
y(a)=y^{\prime}(a)=0, \quad y^{\prime}(b)=\sum_{i=1}^{m-2} b_{i} y\left(\xi_{i}\right),
\end{array}\right.
$$

has a nontrivial solution, where $q$ is a real and continuous function, then

$$
\begin{aligned}
& \int_{a}^{b}|q(s)| d s \\
\geq & \frac{\Gamma(\alpha)}{1+\sum_{i=1}^{m-2} b_{i} T(b)} \cdot \frac{(\alpha-1)^{\alpha-1}}{(b-a)^{\alpha-1}(\alpha-2)^{\alpha-2}} \\
= & \frac{(\alpha-\beta-1)(b-a)^{\alpha-\beta-2}-\sum_{i=1}^{m-2} b_{i}\left(\xi_{i}-a\right)^{\alpha-\beta-1}}{(\alpha-\beta-1)(b-a)^{\alpha-\beta-2}-\sum_{i=1}^{m-2} b_{i}\left(\xi_{i}-a\right)^{\alpha-\beta-1}+\sum_{i=1}^{m-2} b_{i}(b-a)^{\alpha-1}} \\
& \times \frac{\Gamma(\alpha)(\alpha-1)^{\alpha-1}}{(b-a)^{\alpha-1}(\alpha-2)^{\alpha-2}} .
\end{aligned}
$$

\section{Lyapunov inequalities for fractional p-Laplacian boundary value problems}

In this section we present Lyapunov-type inequalities for fractional $p$-Laplacian boundary value problems.

In 2016, Al Arifi et al. [42] considered the nonlinear fractional boundary value problem

$$
\left\{\begin{array}{l}
D^{\beta}\left(\Phi_{p}\left(\left(D^{\alpha} y(t)\right)\right)+q(t) \Phi_{p}(y(t))=0, \quad a<t<b,\right. \\
y(a)=y^{\prime}(a)=y^{\prime}(b)=0, \quad D^{\alpha} y(a)=D^{\alpha} y(b)=0,
\end{array}\right.
$$

where $2<\alpha \leq 3,1<\beta \leq 2, D^{\alpha}, D^{\beta}$ are the Riemann-Liouville fractional derivatives of orders $\alpha$ and $\beta$ respectively, $\Phi_{p}(s)=|s|^{p-2} s, p>1$ is $p$-Laplacian operator and $q:[a, b] \rightarrow \mathbb{R}$ is a continuous function.

For $h \in C([a, b], \mathbb{R})$, the linear variant of the problem 997 :

$$
\left\{\begin{array}{l}
D^{\beta}\left(\Phi_{p}\left(\left(D^{\alpha} y(t)\right)\right)+h(t)=0, \quad a<t<b,\right. \\
y(a)=y^{\prime}(a)=y^{\prime}(b)=0, \quad D^{\alpha} y(a)=D^{\alpha} y(b)=0,
\end{array}\right.
$$

has the unique solution

$$
y(t)=\int_{a}^{b} G(t, s) \Phi_{q}\left(\int_{a}^{b} H(s, \tau) h(\tau) d \tau\right) d s,
$$

where 


$$
H(t, s)=\frac{1}{\Gamma(\beta)} \begin{cases}\left(\frac{b-s}{b-a}\right)^{\beta-1}(t-a)^{\beta-1}, & a \leq t \leq s \leq b, \\ \left(\frac{b-s}{b-a}\right)^{\beta-1}(t-a)^{\beta-1}-(t-s)^{\beta-1}, & a \leq s \leq t \leq b,\end{cases}
$$

and $G(t, s)$ is the Green's function for the boundary value problem

$$
\left\{\begin{array}{l}
D^{\beta} y(t)+h(t)=0,2<\alpha \leq 3, a<t<b \\
y(a)=y^{\prime}(a)=y^{\prime}(b)=0
\end{array}\right.
$$

which is given by

$$
G(t, s)=\frac{1}{\Gamma(\alpha)} \begin{cases}\left(\frac{b-s}{b-a}\right)^{\alpha-2}(t-a)^{\alpha-1}, & a \leq t \leq s \leq b, \\ \left(\frac{b-s}{b-a}\right)^{\alpha-2}(t-a)^{\alpha-1}-(t-s)^{\alpha-1}, & a \leq s \leq t \leq b .\end{cases}
$$

Observe that the following estimates hold:

(i) $0 \leq G(t, s) \leq G(b, s), \quad(t, s) \in[a, b] \times[a, b]$,

(ii) $0 \leq H(t, s) \leq H(s, s), \quad(t, s) \in[a, b] \times[a, b]$.

For the problem (99), the Lyapunov-type inequality is the following:

Theorem 29. Let $2<\alpha \leq 3,1<\beta \leq 2, p>1$, and $q \in C[a, b]$. If (99) has a nontrivial solution, then

$$
\int_{a}^{b}(b-s)^{\beta-1}(s-a)^{\beta-1}|q(s)| d s \geq[\Gamma(\alpha)]^{p-1} \Gamma(\beta)(b-a)^{\beta-1}\left(\int_{a}^{b}(b-s)^{\alpha-2}(s-a) d s\right)^{1-p} .
$$

Now we present an application of this result to eigenvalue problems.

Corollary 16. Let $\lambda$ be an eigenvalue of the problem

$$
\left\{\begin{array}{l}
D_{0}^{\beta}\left(\Phi_{p}\left(D_{0+}^{\alpha} y(t)\right)\right)+\lambda \Phi_{p}(y(t))=0, \quad 0<t<1, \\
y(0)=y^{\prime}(0)=y^{\prime}(1)=0, D_{0+}^{\alpha} y(0)=D_{0+}^{\alpha} y(1)=0
\end{array}\right.
$$

where $2<\alpha \leq 3,1<\beta \leq 2, p>1$, then

$$
|\lambda| \geq \frac{\Gamma(2 \beta)}{\Gamma(\beta)}\left(\frac{\Gamma(\alpha) \Gamma(\alpha+1)}{\Gamma(\alpha-1)}\right)^{p-1} .
$$

In particular, for $p=2$, that is, for $\Phi_{p}(y(t))=y(t)$, the bound on $\lambda$ takes the form:

$$
|\lambda| \geq \frac{\Gamma(\alpha) \Gamma(\alpha+1) \Gamma(2 \beta)}{\Gamma(\alpha-1) \Gamma(\beta)} .
$$

In 2017, Liu et al. [43] considered the nonlinear fractional $p$-Laplacian boundary value problem of the form: 


$$
\left\{\begin{array}{l}
D^{\beta}\left(\Phi_{p}\left({ }^{C} D^{\alpha} y(t)\right)\right)-q(t) f(y(t))=0, \quad a<t<b, \\
y^{\prime}(a)={ }^{C} D^{\alpha} y(a)=0, \quad y(b)={ }^{C} D^{\alpha} y(b)=0,
\end{array}\right.
$$

where $1<\alpha, \beta \leq 2,{ }^{C} D^{\alpha}, D^{\beta}$ are the Riemann-Liouville fractional derivatives of orders $\alpha$ and $\beta$ respectively, $\Phi_{p}(s)=|s|^{p-2} s, p>1$, and $q:[a, b] \rightarrow \mathbb{R}$ is a continuous function.

An integral equation equivalent to the problem (106) is

$$
y(t)=\int_{a}^{b} G(t, s) \Phi_{q}\left(\int_{a}^{b} H(s, \tau) f(y(\tau)) d \tau\right) d s,
$$

where

$$
G(t, s)=\frac{1}{\Gamma(\alpha)} \begin{cases}(b-s)^{\alpha-1}, & a \leq t \leq s \leq b, \\ (b-s)^{\alpha-1}-(t-s)^{\alpha-1}, & a \leq s \leq t \leq b,\end{cases}
$$

and

$$
H(t, s)=\frac{1}{\Gamma(\beta)} \begin{cases}\left(\frac{s-a}{b-a}\right)^{\beta-1}(b-s)^{\beta-1}, & a \leq t \leq s \leq b, \\ \left(\frac{s-a}{b-a}\right)^{\beta-1}(b-s)^{\beta-1}-(t-s)^{\beta-1}, & a \leq s \leq t \leq b .\end{cases}
$$

Moreover, the following estimates hold:

(i) $H(t, s) \geq 0$ for all $a \leq t, s \leq b$;

(ii) $\max _{t \in[a, b]} H(t, s)=H(s, s), s \in[a, b]$;

(iii) $H(t, s)$ has a unique maximum given by

$$
\max _{s \in[a, b]} H(s, s)=\frac{(b-a)^{\beta-1}}{4^{\beta-1} \Gamma(\beta)} ;
$$

(iv) $0 \leq G(t, s) \leq G(s, s)=\frac{1}{\Gamma(\alpha)}(b-s)^{\alpha-1}$ for all $a \leq t, s \leq b$;

(v) $G(t, s)$ has a unique maximum given by

$$
\max _{s \in[a, b]} G(s, s)=\frac{1}{\Gamma(\alpha)}(b-a)^{\alpha-1} .
$$

The Lyapunov-type inequalities for the problem (106) are as follows.

Theorem 30. Let $p:[a, b] \rightarrow \mathbb{R}^{+}$be a real Lebesgue function. Suppose that there exists a positive constant $M$ satisfying $0 \leq f(x) \leq M \Phi_{p}(x)$ for any $x \in \mathbb{R}^{+}$. If (106) has a nontrivial solution in $E^{+}=\{y \in C[a, b], y(t) \geq 0$, for any $t \in[a, b]$ and $\|y\| \neq$ $0\}$, then the following Lyapunov inequality holds:

$$
\int_{a}^{b} q(s) d s>\frac{4^{\beta-1} \Gamma(\beta)}{M(b-a)^{\beta-1}} \Phi_{p}\left(\frac{\Gamma(\alpha+1)}{(b-a)^{\alpha}}\right) .
$$


Theorem 31. Let $p:[a, b] \rightarrow \mathbb{R}^{+}$be a real Lebesgue function. Assume that $f \in$ $C\left(\mathbb{R}^{+}, \mathbb{R}^{+}\right)$is a concave and nondecreasing function. If $(106)$ has a nontrivial solution in $E^{+}=\{y \in C[a, b], y(t) \geq 0$, for any $t \in[a, b]$ and $\|y\| \neq 0\}$, then the following Lyapunov inequality holds:

$$
\int_{a}^{b} q(s) d s>\frac{4^{\beta-1} \Gamma(\beta) \Phi_{p}(\Gamma(\alpha+1)) \Phi_{p}(\eta)}{M(b-a)^{\alpha p+\beta-\alpha-1} f(\eta)},
$$

where $\eta=\max _{t \in[a, b]} y(t)$.

As an application of the foregoing results, we give the following corollary.

Corollary 17. If $\lambda \in\left[0,4^{\beta-1} \Gamma(\beta) \Phi_{p}(\Gamma(\alpha+1))\right]$, then the following eigenvalue problem

$$
\left\{\begin{array}{l}
D^{\beta}\left(\Phi_{p}\left({ }^{C} D_{0+}^{\alpha} y(t)\right)\right)-\lambda \Phi_{p}(y(t))=0, \quad 0<t<1, \\
y^{\prime}(0)={ }^{C} D^{\alpha}=0, \quad y(1)={ }^{C} D^{\alpha} D^{\alpha} y(0)=0,
\end{array}\right.
$$

has no corresponding eigenfunction $y \in E^{+}$, where $1<\alpha, \beta \leq 2$, and $p>1$.

\section{Lyapunov inequalities for boundary value problems with mixed fractional derivatives}

In 2017, Guezane-Lakoud et al. [44] obtained a Lyapunov-type inequality for the following problem involving both right Caputo and left Riemann-Liouville fractional derivatives:

$$
\left\{\begin{array}{l}
-{ }^{C} D_{b-}^{\alpha} D_{a+}^{\beta} y(t)+q(t) y(t), t \in[a, b], \\
y(a)=D_{a+}^{\beta} y(b)=0,
\end{array}\right.
$$

where $0<\alpha, \beta<1,1<\alpha+\beta \leq 2,{ }^{C} D_{b-}^{\alpha}$ denotes the right Caputo fractional derivative, $D_{a+}^{\beta}$ denotes left Riemann-Liouville fractional derivative, and $q:[a, b] \rightarrow \mathbb{R}^{+}$ is a continuous function.

The left and right Riemann-Liouville fractional integrals of order $p>0$ for a function $g:(0, \infty) \rightarrow \mathbb{R}$ are respectively defined by

$$
\begin{aligned}
& I_{a+}^{p} g(t)=\int_{a}^{t} \frac{(t-s)^{p-1}}{\Gamma(p)} g(s) d s, \\
& I_{b-}^{p} g(t)=\int_{t}^{b} \frac{(s-t)^{p-1}}{\Gamma(p)} g(s) d s,
\end{aligned}
$$

provided the right-hand sides are point-wise defined on $(0, \infty)$, where $\Gamma$ is the Gamma function. 
The left Riemann-Liouville fractional derivative and the right Caputo fractional derivative of order $p>0$ for a continuous function $g:(0, \infty) \rightarrow \mathbb{R}$ are respectively given by

$$
\begin{gathered}
D_{a+}^{p} g(t)=\frac{d^{n}}{d t^{n}}\left(I_{a+}^{n-p}\right)(t), \\
{ }^{c} D_{b-}^{p} g(t)=(-1)^{n} I_{b-}^{n-p} g^{(n)}(t),
\end{gathered}
$$

where $n-1<p<n$.

The function $y \in C[a, b]$ is a solution to the problem (111) if and only if $y$ satisfies the integral equation

$$
y(t)=\int_{a}^{b} G(t, s) q(s) f(y(s)) d s,
$$

where $G(t, s)$ is the Green's function defined by

$$
G(t, s)=\frac{1}{\Gamma(\alpha) \Gamma(\beta)} \begin{cases}\int_{a}^{r}(t-s)^{\beta-1}(r-s)^{\alpha-12}, & a \leq r \leq t \leq b, \\ \int_{a}^{t}(t-s)^{\beta-1}(r-s)^{\alpha-1}, & a \leq t \leq s \leq b .\end{cases}
$$

The above Green's function satisfies the following properties:

(i) $G(t, s) \geq 0$, for all $a \leq r \leq t \leq b$;

(ii) $\max _{t \in[a, b]} G(t, r)=G(r, r)$ for all $r \in[a, b]$;

(iii) $\max _{r \in[a, b]} G(r, r)=\frac{(b-a)^{\alpha+\beta-1}}{(\alpha+\beta-1) \Gamma(\alpha) \Gamma(\beta)}$.

The following result describes the Lyapunov inequality for problem 1111).

Theorem 32. Assume that $0<\alpha, \beta<1$ and $1<\alpha+\beta \leq 2$. If the fractional boundary value problem (111) has a nontrivial continuous solution, then

$$
\int_{a}^{b}|q(r)| d r \geq \frac{(\alpha+\beta-1) \Gamma(\alpha) \Gamma(\beta)}{(b-a)^{\alpha+\beta-1}} .
$$

\section{Lyapunov inequality for Hadamard type fractional boundary value problems}

Let us begin this section with some fundamental definitions.

Definition 4. [14] The Hadamard derivative of fractional order $q$ for a function $g$ : $[1, \infty) \rightarrow \mathbb{R}$ is defined as

${ }^{H} D^{q} g(t)=\frac{1}{\Gamma(n-q)}\left(t \frac{d}{d t}\right)^{n} \int_{1}^{t}\left(\log \frac{t}{s}\right)^{n-q-1} \frac{g(s)}{s} d s, n-1<q<n, n=[q]+1$, 
where $[q]$ denotes the integer part of the real number $q$ and $\log (\cdot)=\log _{e}(\cdot)$ is the usual Napier logarithm.

Definition 5. [14] The Hadamard fractional integral of order $q$ for a function $g$ is defined as

$$
I^{q} g(t)=\frac{1}{\Gamma(q)} \int_{1}^{t}\left(\log \frac{t}{s}\right)^{q-1} \frac{g(s)}{s} d s, q>0,
$$

provided the integral exists.

In 2017, Ma et al. [45] obtained a Lyapunov-type inequality for a Hadamard fractional boundary value problem

$$
\left\{\begin{array}{l}
{ }^{H} D^{\alpha} y(t)-q(t) y(t)=0, \quad 1<t<e, \\
y(1)=y(e)=0
\end{array}\right.
$$

where ${ }^{H} D^{\alpha}$ is the fractional derivative in the sense of the Hadamard of order $1<$ $\alpha \leq 2$ and $q:[a, b] \rightarrow \mathbb{R}$ is a continuous function.

The function $y \in C([1, e], \mathbb{R})$ is a solution of the boundary value problem $[113)$ if and only if $y$ satisfies the integral equation

$$
y(t)=\int_{a}^{b} G(t, s) q(s) y(s) d s,
$$

where $G(t, s)$ is the Green's function defined by

$$
G(t, s)=\frac{1}{\Gamma(\alpha)} \begin{cases}-\left(\log \frac{e}{s}\right)^{\alpha-1} \frac{(\log t)^{\alpha-1}}{s}, & 1 \leq t \leq s \leq e, \\ -\left(\log \frac{e}{s}\right)^{\alpha-1} \frac{(\log t)^{\alpha-1}}{s}+\left(\log \frac{t}{s}\right)^{\alpha-1} \frac{1}{s}, & 1 \leq s \leq t \leq e .\end{cases}
$$

such that

$$
|G(t, s)| \leq \frac{\lambda^{\alpha-1}}{\Gamma(\alpha)}(1-\lambda)^{\alpha-1} \exp (-\lambda),
$$

with

$$
\lambda=\frac{1}{2}\left(2 \alpha-1-\sqrt{(2 \alpha-2)^{2}+1}\right) .
$$

The result concerning the Lyapunov-type inequality for the problem (113) is as follows.

Theorem 33. If there exists a nontrivial continuous solution of the fractional boundary value problem (113), then

$$
\int_{a}^{b}|q(s)| d s>\Gamma(\alpha) \lambda^{1-\alpha} \cdot(1-\lambda)^{1-\alpha} \exp \lambda,
$$

where $\lambda$ is defined by (116).

For recent results on Hadamard type fractional boundary value problems, we refer the interested reader to the book [46]. 


\section{Lyapunov inequality for boundary value problems with Prabhakar fractional derivative}

In [47], the authors discussed Lyapunov-type inequality for the following fractional boundary value problem involving the $k$-Prabhakar derivative:

$$
\left\{\begin{array}{l}
\left({ }_{k} D_{\rho, \beta, \omega, a+}^{\gamma} y\right)(t)+q(t) f(y(t))=0, \quad a<t<b, \\
y(a)=y(b)=0,
\end{array}\right.
$$

where ${ }_{k} D_{\rho, \beta, \omega, a+}^{\gamma}$ is the $k$-Prabhakar differential operator of order $\beta \in(1,2], k \in \mathbb{R}^{+}$ and $\rho, \gamma, \omega \in \mathbb{C}$. The $k$-Prabhakar integral operator is defined as

$$
\left({ }_{k} P_{\alpha, \beta, \omega} \phi\right)(t)=\int_{0}^{x} \frac{(x-t)^{\frac{\beta}{k}-1}}{k} E_{k, \alpha, \beta}\left[\omega(x-t)^{\frac{\alpha}{k}}\right] \phi(t) d t, x>0,
$$

where $E_{k, \alpha, \beta}$ is the $k$-Mittag-Leffler function given by

$$
E_{k, \alpha, \beta}^{\gamma}(z)=\sum_{n=0}^{\infty} \frac{(\gamma)_{n, k} z^{n}}{\Gamma_{k}(\alpha n+\beta) n !}
$$

$\Gamma_{k}(x)$ is the $k$-Gamma function $\Gamma_{k}(x)=\lim _{n \rightarrow \infty} \frac{n ! k^{n}(n k)^{\frac{x}{k}-1}}{(x)_{n, k}}$ and $(\gamma)_{n, k}=\frac{\Gamma_{k}(\gamma+n k)}{\Gamma_{k}(\gamma)}$ is the Pochhammer $k$-symbol.

The $k$-Prabhakar derivative is defined as

$$
{ }_{k} D_{\rho, \beta, \omega}^{\gamma} f(x)=\left(\frac{d}{d x}\right)^{m} k_{k}^{m} P_{\rho, m k-\beta, \omega}^{-\gamma} f(x),
$$

where $m=[\beta / k]+1$.

An integral equation related to the problem (118) is

$$
y(t)=\int_{a}^{b} G(t, s) q(s) y(s) d s,
$$

where

$$
G(t, s)= \begin{cases}\frac{(t-a)^{\frac{\beta}{k}-1} E_{k, \rho, \beta}^{\gamma}\left(\omega(t-a)^{\frac{\rho}{k}}\right)(b-s)^{\frac{\beta}{k}-1}}{(b-a)^{\frac{\beta}{k}-1} E_{k, \rho, \beta}^{\gamma}\left(\omega(b-a)^{\frac{\rho}{k}}\right) k} E_{k, \rho, \beta}^{\gamma}\left(\omega(b-s)^{\frac{\rho}{k}}\right), & a \leq t \leq s \leq b, \\ \frac{(t-a)^{\frac{\beta}{k}-1} E_{k, \rho, \beta}^{\gamma}\left(\omega(t-a)^{\frac{\rho}{k}}\right)(b-s)^{\frac{\beta}{k}-1}}{(b-a)^{\frac{\beta}{k}-1} E_{k, \rho, \beta}^{\gamma}\left(\omega(b-a)^{\frac{\rho}{k}}\right) k} E_{k, \rho, \beta}^{\gamma}\left(\omega(b-s)^{\frac{\rho}{k}}\right. & \\ -\frac{(t-s) \frac{\beta}{k}-1}{k} E_{k, \rho, \beta}^{\gamma}\left(\omega(t-s)^{\frac{\rho}{k}}\right), & a \leq s \leq t \leq b,\end{cases}
$$

which satisfies the following properties:

(i) $G(t, s) \geq 0$ for all $a \leq t, s \leq b$; 
(ii) $\max _{t \in[a, b]} G(t, s)=G(s, s)$ for all $s \in[a, b]$;

(iii) $G(t, s)$ has a unique maximum given by

$$
\max _{s \in[a, b]} G(s, s)=\left(\frac{b-a}{4}\right)^{\frac{\beta}{k}-1} \frac{E_{k, \rho, \beta}^{\gamma}\left(\omega\left(\frac{b-a}{2}\right)^{\frac{\rho}{k}}\right) E_{k, \rho, \beta}^{\gamma}\left(\omega\left(\frac{b-a}{2}\right)^{\frac{\rho}{k}}\right)}{k E_{k, \rho, \beta}^{\gamma}\left(\omega(b-a)^{\frac{\rho}{k}}\right)} .
$$

The Lyapunov-type inequality for the problem 1118 is given in the following result.

Theorem 34. If the problem (118) has a nontrivial solution, then

$$
\int_{a}^{b}|q(s)| d s \geq\left(\frac{4}{b-a}\right)^{\frac{\beta}{k}-1} \frac{k E_{k, \rho, \beta}^{\gamma}\left(\omega(b-a)^{\frac{\rho}{k}}\right)}{E_{k, \rho, \beta}^{\gamma}\left(\omega\left(\frac{b-a}{2}\right)^{\frac{\rho}{k}}\right) E_{k, \rho, \beta}^{\gamma}\left(\omega\left(\frac{b-a}{2}\right)^{\frac{\rho}{k}}\right)} .
$$

The special case $k=1$ for the problem (118) has recently been studied in [48].

In 2017, Pachpatte et al. [49] established some Lyapunov-type inequalities for the following hybrid fractional boundary value problem

$$
\left\{\begin{array}{l}
D_{\rho, \beta, \omega}^{\gamma}\left[\frac{y(t)}{f(t, y(t))}-\sum_{i=1}^{n} E_{\rho, \beta, \omega}^{\gamma} h_{i}(t, y(t))\right]+g(t) y(t)=0, \quad t \in(a, b), \\
y(a)=y(b)=0
\end{array}\right.
$$

where $D_{\rho, \beta, \omega}^{\gamma}$ denotes the Prabhakar fractional derivative of order $\beta \in(1,2]$ starting from a point $a, y \in C([a, b], \mathbb{R}), g \in L^{1}((a, b], \mathbb{R}), f \in C^{1}([a, b] \times \mathbb{R}, \mathbb{R} \backslash\{0\}), h_{i} \in$ $C([a, b] \times \mathbb{R}, \mathbb{R}), \forall i=1,2, \ldots, n$ and $E_{\rho, \mu, \omega}^{\alpha}$ is the Prabhakar fractional integral of order $\mu$ with the lower limit at the point $a$.

We consider two cases: (I) $h_{i}=0, i=1,2, \ldots, n$ and (II) $h_{i} \neq 0, i=1,2, \ldots, n$. Case I: $h_{i}=0, i=1,2, \ldots, n$. We consider the problem (120) with $h_{i}(t, \cdot)=0$ for all $t \in[a, b]$. For $\alpha \in(2,3]$, we first construct a Green's function for the following boundary value problem

$$
\left\{\begin{array}{l}
D_{\rho, \mu, \omega}^{\alpha}\left[\frac{y(t)}{f(t, y(t))}\right]+g(t) y(t)=0, \quad t \in(a, b), \\
y(a)=y(b)=0
\end{array}\right.
$$

with the assumption that $f$ is continuously differentiable and $f(t, y(t)) \neq 0$ for all $t \in[a, b]$. Let $y \in A C([a, b], \mathbb{R})$ be a solution of the problem [121). Then the function $y$ satisfies the following integral equation

$$
y=f(t, y) \int_{a}^{b} G(t, s) g(s) y(s) d s,
$$


where $G(t, s)$ is the Green's function defined by (119). The Lyapunov-type inequality for this case is as follows.

Theorem 35. If the problem (121) has a nontrivial solution, then

$$
\int_{a}^{b}|q(s)| d s \geq \frac{1}{\|f\|}\left(\frac{4}{b-a}\right)^{\beta-1} \frac{E_{\rho, \beta}^{\gamma}\left(\omega(b-a)^{\rho}\right)}{E_{\rho, \beta}^{\gamma}\left(\omega\left(\frac{b-a}{2}\right)^{\rho}\right) E_{\rho, \beta}^{\gamma}\left(\omega\left(\frac{b-a}{2}\right)^{\rho}\right)},
$$

where $\|f\|=\sup _{t \in[a, b], y \in \mathbb{R}}|f(t, y)|$.

Case II: $h_{i} \neq 0, i=1,2, \ldots, n$. Let $y \in A C[a, b]$ be a solution of the problem (120) given by

$$
y(t)=f(t, y(t)) \int_{a}^{b} G(t, s) g\left[(s) y(s)-\sum_{i=1}^{n} \int_{a}^{b} h_{i}(s, y(s))\right] d s,
$$

where $G(t, s)$ is defined as in 119 .

Theorem 36. (Lyapunov-type inequality) Assume that $\left|q(t) y(t)-\sum_{i=1}^{n} h_{i}(t, y(t))\right| \leq$ $K \mid q t) \mid\|y\|, K \in \mathbb{R}$. If a nontrivial solution for the problem (120) exists, then

$$
\int_{a}^{b}|q(s)| d s \geq \frac{1}{K\|f\|}\left(\frac{4}{b-a}\right)^{\beta-1} \frac{E_{\rho, \beta}^{\gamma}\left(\omega(b-a)^{\rho}\right)}{E_{\rho, \beta}^{\gamma}\left(\omega\left(\frac{b-a}{2}\right)^{\rho}\right) E_{\rho, \beta}^{\gamma}\left(\omega\left(\frac{b-a}{2}\right)^{\rho}\right)} .
$$

\section{Lyapunov inequality for $q$-difference boundary value problems}

Let a $q$-real number denoted by $[a]_{q}$ be defined by

$$
[a]_{q}=\frac{1-q^{a}}{1-q}, a \in \mathbb{R}, q \in \mathbb{R}^{+} \backslash\{1\} .
$$

The $q$-analogue of the Pochhammer symbol ( $q$-shifted factorial) is defined as

$$
(a ; q)_{0}=1, \quad(a ; q)_{k}=\prod_{i=0}^{k-1}\left(1-a q^{i}\right), k \in \mathbb{N} \cup\{\infty\} .
$$

The $q$-analogue of the exponent $(x-y)^{k}$ is

$$
(x-y)_{a}^{(0)}=1, \quad(x-y)_{a}^{(k)}=\prod_{j=0}^{k-1}\left((x-a)-(y-a) q^{j}\right), k \in \mathbb{N}, x, y \in \mathbb{R} .
$$

More generally, if $\gamma \in \mathbb{R}$, then 


$$
(x-y)_{a}^{(\gamma)}=(x-a)^{\gamma} \prod_{i=0}^{\infty} \frac{(x-a)-q^{i}(y-a)}{(x-a)-q^{\gamma+1}(y-a)} .
$$

The $q$-Gamma function $\Gamma_{q}(y)$ is defined as

$$
\Gamma_{q}(y)=\frac{(1-q)_{0}^{(y-1)}}{(1-q)^{y-1}}
$$

where $y \in \mathbb{R} \backslash\{0,-1,-2, \ldots\}$. Observe that $\Gamma_{q}(y+1)=[y]_{q} \Gamma_{q}(y)$.

The $q$-derivative of a function $f:[a ; b] \rightarrow \mathbb{R}(a<b)$ is defined by

$$
\left({ }_{a} D_{q} f\right)(t)=\frac{f(t)-f(q t+(1-q) a}{(1-q)(t-a)}, t \neq a
$$

and

$$
\left({ }_{a} D_{q} f\right)(a)=\lim _{t \rightarrow a}\left({ }_{a} D_{q} f\right)(t) .
$$

In 2016, Jleli and Samet [50] established a Lyapunov-type inequality for a Dirichlet boundary value problem of fractional $q$-difference equations given by

$$
\left\{\begin{array}{l}
\left({ }_{a} D^{\alpha} y\right)(t)+\phi(t) y(t)=0, \quad a<t<b, q \in[0,1), 1<\alpha \leq 2, \\
y(a)=y(b)=0
\end{array}\right.
$$

where ${ }_{a} D^{\alpha}$ denotes the fractional $q$-derivative of Riemann-Liouville type and $\phi$ : $[a, b] \rightarrow \mathbb{R}$ is a continuous function.

The solution $y \in C([a, b], \mathbb{R})$ of the problem (124) satisfies the integral equation

$y(t)=\int_{a}^{t} G_{1}(t, q s+(1-q) a) \phi(s) y(s)_{a} d_{q} s+\int_{t}^{b} G_{2}(t, s) \phi(s) y(s)_{a} d_{q} s, a \leq t \leq b$,

where

$$
\begin{gathered}
G_{1}(t, s)=\frac{1}{\Gamma_{q}(\alpha)}\left(\frac{(t-a)^{\alpha-1}}{(b-a)^{\alpha-1}}(b-s)_{a}^{(\alpha-1)}-(t-s)_{a}^{(\alpha-1)}\right), a \leq s \leq t \leq b, \\
G_{2}(t, s)=\frac{(t-a)^{\alpha-1}}{\Gamma_{q}(\beta)(b-a)^{\alpha-1}}\left(b-(q s+(1-q) a)_{a}^{(\alpha-1)}, a \leq t \leq s \leq b,\right.
\end{gathered}
$$

satisfying the relations

(i) $0 \leq G_{1}(t, q s+(1-q) a) \leq G_{2}(s, s), a<s \leq t \leq b$;

(ii) $G_{2}(a, s)=0 \leq G_{2}(t, s) \leq G_{2}(s, s), a \leq t \leq s \leq b$.

Theorem 37. (Lyapunov-type inequality) If the problem (124) has a nontrivial solution, then

$$
\int_{a}^{b}(s-a)^{\alpha-1}(b-(q s+(1-q) a))_{a}^{(\alpha-1)}|\phi(s)|{ }_{a} d_{q} s \geq \Gamma_{q}(\alpha)(b-a)^{\alpha-1} .
$$


Taking $\alpha=2$ in the above theorem we have the following corollary.

Corollary 18. If a nontrivial continuous solution to the q-difference boundary value problem,

$$
\left\{\begin{array}{l}
\left({ }_{a} D_{q}^{2} y\right)(t)+\phi(t) y(t)=0, \quad a<t<b, q \in(0,1), \\
y(a)=y(b)=0,
\end{array}\right.
$$

exists, where $\phi:[a, b] \rightarrow \mathbb{R}$ is a continuous function, then

$$
\int_{a}^{b}(s-a)(b-(q s+(1-q) a))|\phi(s)|{ }_{a} d_{q} s \geq(b-a) .
$$

Some recent work on $q$-difference boundary value problems can be found in [51].

\section{Lyapunov inequality for boundary value problems involving a fractional derivative with respect to a certain function}

In 2017, Jleli et al. [52] considered the following fractional boundary value problem involving a fractional derivative with respect to a certain function $g$

$$
\left\{\begin{array}{l}
\left(D_{a, g}^{\alpha} y\right)(t)+q(t) y(t)=0, \quad a<t<b, 1<\alpha \leq 2, \\
y(a)=y(b)=0
\end{array}\right.
$$

where $D_{a, g}^{\alpha}$ is the fractional derivative operator of order $\alpha$ with respect to a nondecreasing function $g \in C^{1}([a, b], \mathbb{R})$ with $g^{\prime}(x)>0$, for all $x \in[a, b]$, and $q:[a, b] \rightarrow \mathbb{R}$ is a continuous function.

Let $f \in L^{1}((a, b), \mathbb{R})$. The fractional integral of order $\alpha>0$ of $f$ with respect to the function $g$ is defined by

$$
\left(I_{a, g}^{\alpha} f\right)(t)=\frac{1}{\Gamma(\alpha)} \int_{a}^{t} \frac{g^{\prime}(s) f(s)}{(g(t)-g(s))^{1-\alpha}} d s, \text { a.e. } t \in[a, b] .
$$

Let $\alpha>0$ and $n$ be the smallest integer greater than or equal to $\alpha$. Let $f:[a, b] \rightarrow \mathbb{R}$ be a function such that $\left(\left(1 / g^{\prime}(t)\right)(d / d t)^{n} I_{a, g}^{n-\alpha} f\right.$ exists almost everywhere on $[a, b]$. Then the fractional derivative of order $\alpha$ of $f$ with respect to the function $g$ is defined as

$$
\begin{aligned}
D_{a, g}^{\alpha} f(t) & =\left(\frac{1}{g^{\prime}(t)} \frac{t}{d t}\right)^{n} I_{a, g}^{n-\alpha} f(t) \\
& =\frac{1}{\Gamma(n-\alpha)}\left(\frac{1}{g^{\prime}(t)} \frac{t}{d t}\right)^{n} \int_{a}^{t} \frac{g^{\prime}(s) f(s)}{(g(t)-g(s))^{\alpha-n+1}} d s, \text { for a.e } t \in[a, b] .
\end{aligned}
$$

Theorem 38. Assume that $q \in C([a, b], \mathbb{R})$ and $g \in C^{1}([a, b], \mathbb{R})$ be a nondecreasing function with $g^{\prime}(x)>0$, for all $x \in[a, b]$. If the problem (126) has a nontrivial solution, then 


$$
\int_{a}^{b}\left[\left(g(s)-g(a)((g(b)-g(s))]^{\alpha-1} g^{\prime}(s)|q(s)| d s \geq \Gamma(\alpha)(g(b)-g(a))^{\alpha-1} .\right.\right.
$$

From inequality (127), we can obtain Lyapunov-type inequalities for different choices of the function $g$. For instance, for $g(x)=x^{\beta}, x \in[a, b]$ and $g(x)=\log x, x \in$ $[a, b]$ we have respectively the following results.

Corollary 19. If the problem (126) has a nontrivial solution and $g(x)=x^{\beta}, x \in$ $[a, b], 0<a<b$, then

$$
\int_{a}^{b}|q(s)| d s \geq \frac{\Gamma(\alpha)\left(b^{\beta}-a^{\beta}\right)^{\alpha-1}}{\beta \phi_{\alpha, \beta}\left(s^{*}(\alpha, \beta)\right)}
$$

where $\phi_{\alpha, \beta}\left(s^{*}(\alpha, \beta)\right)=\max \left\{\phi_{\alpha, \beta}(s): s \in[a, b]\right\}>0$.

Taking $g(x)=\log x, x \in[a, b], 0<a<b$, in Theorem 38, we deduce the following Hartman-Wintner-type inequality

$$
\int_{a}^{b}\left[\left(\log \frac{s}{a}\right)\left(\log \frac{b}{s}\right)\right]^{\alpha-1} \frac{|q(s)|}{s} d s \geq \Gamma(\alpha)\left(\log \frac{b}{a}\right)^{\alpha-1},
$$

for the Hadamard fractional boundary value problem of the form:

$$
\left\{\begin{array}{l}
\left({ }^{H} D_{a}^{\alpha} y\right)(t)+q(t) y(t)=0, \quad a<t<b, 1<\alpha \leq 2, \\
y(a)=y(b)=0 .
\end{array}\right.
$$

In order to demonstrate the application of Theorem 38 we consider the eigenvalue problem

$$
\left\{\begin{array}{l}
D_{a, g}^{\alpha} y(t)+\lambda y(t)=0, \quad a<t<b \\
y(a)=y(b)=0
\end{array}\right.
$$

and use the Lyapunov-type inequality (127) to obtain the following result.

Theorem 39. If $\lambda$ is an eigenvalue of fractional boundary value problem (129), then the following inequality holds

$$
|\lambda| \geq \frac{\Gamma(\alpha)(g(b)-g(a))^{\alpha-1}}{\int_{g(a)}^{g(b)}(x-g(a))^{\alpha-1}(g(b)-x)^{\alpha-1} d x} .
$$

\section{Lyapunov inequality for boundary value problems involving left and right derivatives}

The left and right Caputo fractional derivatives are defined via the RiemannLiouville fractional derivatives (see [14 p. 91]). In particular, they are defined for a class of absolutely continuous functions. 
Definition 6. (Left and Right Riemann-Liouville Fractional Integrals [14]). Let $f$ be a function defined on $[a, b]$. The left and right Riemann-Liouville fractional integrals of order $\gamma$ for function $f$ denoted by $I_{a+}^{\gamma}$ and $I_{b-}^{\gamma}$ respectively, are defined by

$$
I_{a+}^{\gamma} f(t)=\frac{1}{\Gamma(\gamma)} \int_{a}^{t}(t-s)^{\gamma-1} f(s) d s, t \in[a, b], \gamma>0,
$$

and

$$
I_{b-}^{\gamma} f(t)=\frac{1}{\Gamma(\gamma)} \int_{t}^{b}(t-s)^{\gamma-1} f(s) d s, t \in[a, b], \gamma>0,
$$

provided the right-hand sides are pointwise defined on $[a, b]$, where $\Gamma>0$ is the gamma function.

Definition 7. (Left and Right Riemann-Liouville Fractional Derivatives [14]). Let $f$ be a function defined on $[a, b]$. The left and right Riemann-Liouville fractional derivatives of order $\gamma$ for function $f$ denoted by ${ }_{a} D_{t}^{\gamma} f(t)$ and ${ }_{t} D_{b}^{\gamma} f(t)$, respectively, are defined by

$$
{ }_{a} D_{t}^{\gamma} f(t)=\frac{d^{n}}{d t^{n}} D_{t}^{\gamma-n} f(t)=\frac{1}{\Gamma(n-\gamma)} \frac{d^{n}}{d t^{n}}\left(\int_{a}^{t}(t-s)^{n-\gamma-1} f(s) d s\right)
$$

and

$$
{ }_{t} D_{b}^{\gamma} f(t)=(-1)^{n} \frac{d^{n}}{d t^{n}}{ }_{t} D_{b}^{\gamma-n} f(t)=(-1)^{n} \frac{1}{\Gamma(n-\gamma)} \frac{d^{n}}{d t^{n}}\left(\int_{t}^{b}(t-s)^{n-\gamma-1} f(s) d s\right)
$$

where $t \in[a, b], n-1 \leq \gamma<n$ and $n \in \mathbb{N}$.

In 2017, Chen et al. [53] obtained a Lyapunov-type inequality for the following boundary value problem

$$
\left\{\begin{array}{l}
\frac{d}{d t}\left(\frac{1}{2} I_{a+}^{\beta} y^{\prime}(t)+\frac{1}{2} I_{b-}^{\beta} y^{\prime}(t)\right)+q(t) y(t)=0, a<t<b \\
y(a)=y(b)=0
\end{array}\right.
$$

where $I_{a+}^{\beta}$ and $I_{b-}^{\beta}$ denote the left and right Riemann-Liouville fractional integrals of order $0 \leq \beta<1$, respectively, and $q \in L^{1}([a, b], \mathbb{R})$.

Theorem 40. Let $q \in L^{1}([a, b], \mathbb{R})$ be a nonnegative function and there exists a nontrivial solution for the boundary value problem (130). Then

$$
\int_{a}^{b}|q(s)| d t \geq\left(\frac{2(b-a)^{\alpha-1 / 2}}{\Gamma(\alpha)(2 \alpha-1)^{1 / 2}} \frac{1}{|\cos (\pi \alpha)|^{1 / 2}}\right)^{-2}, \alpha=1-\frac{\beta}{2}
$$

Jleli et al. [54] considered the following quasilinear problem involving both left and right Riemann-Liouville fractional derivative operators: 


$$
\left\{\begin{array}{l}
\frac{1}{2}\left({ }_{t} D_{b}^{\alpha}\left(\left|{ }_{a} D_{t}^{\alpha}\right|{ }_{a}^{p-2} D_{t}^{\alpha} y\right)+{ }_{a} D_{t}^{\alpha}\left(\left|{ }_{t} D_{b}^{\alpha} y\right|_{t}^{p-2} D_{b}^{\alpha} y\right)\right)=q(t)|y|^{p-2} y, \quad a<t<b, \\
y(a)=y(b)=0,
\end{array}\right.
$$

where ${ }_{a} D_{t}^{\alpha}$ and ${ }_{t} D_{b}^{\alpha}$ denote the left Riemann-Liouville fractional derivative of order $\alpha \in(0,1)$ and the right Riemann-Liouville fractional derivative of order $\alpha$ respectively. Note that for $\alpha=1$, problem (131) reduces to the $p$-Laplacian problem

$$
\left\{\begin{array}{l}
\left(\left|y^{\prime}\right|^{p-2} y\right)^{\prime}+q(t)|y|^{p-2} y=0, \quad a<t<b, \quad p>1, \\
y(a)=y(b)=0
\end{array}\right.
$$

The Lyapunov-type inequality for the problem (131) is given in the following theorem.

Theorem 41. Assume that $0 \frac{1}{p}<\alpha<1$ and $q \in L^{1}(a, b)$. If problem (131) admits a nontrivial solution $y \in A C_{a}^{\alpha, p}[a, b] \cap A C_{b}^{\alpha, p}[a, b] \cap C[a, b]$ such that $|y(c)|=\|y\|_{\infty}, c \in$ $(a, b)$, then

$$
\int_{a}^{b} q^{+}(s) d s \geq\left(\frac{2(\alpha p-1)}{p-1}\right)^{p-1} \frac{[\Gamma(\alpha)]^{p}}{\left((c-a)^{\frac{\alpha p-1}{p-1}}+(b-c)^{\frac{\alpha p-1}{p-1}}\right)^{p-1}}
$$

where $q^{+}(t)=\max \{q(t), 0\}$ for $t \in[a, b], A C_{a}^{\alpha, p}[a, b]=\left\{y \in L^{1}(a, b):_{a} D_{t}^{\alpha} y \in\right.$ $\left.L^{p}(a, b)\right\}$ and $A C_{b}^{\alpha, p}[a, b]=\left\{y \in L^{1}(a, b):_{t} D_{b}^{\alpha} y \in L^{p}(a, b)\right\}$.

\section{Lyapunov inequality for boundary value problems with nonsingular Mittag-Leffler kernel}

In 2017, Abdeljawad [55] proved a Lyapunov-type inequality for the following Riemann-Liouville type fractional boundary value problem of order $2<\alpha \leq 3$ in terms of Mittag-Leffler kernels:

$$
\left\{\begin{array}{l}
\left({ }_{a}^{A B R} D^{\alpha} y\right)(t)+q(t) y(t)=0, \quad a<t<b, 2<\alpha \leq 3, \\
y(a)=y(b)=0
\end{array}\right.
$$

where ${ }_{a}^{A B R} D^{\alpha}$ denotes the left Riemann-Liouville fractional derivative defined by

$$
\left({ }_{a}^{A B R} D^{\alpha} f\right)(t)=\frac{B(\alpha)}{1-\alpha} \frac{d}{d t} \int_{a}^{t} f(x) E_{\alpha}\left(-\alpha \frac{(t-x)^{\alpha}}{1-\alpha}\right) d s,
$$

where $B(\alpha)$ is a normalization function such that $B(0)=B(1)=1$, and $E_{\alpha}$ is the generalized Mittag-Leffler function given by $E_{\alpha}\left(-t^{\alpha}\right)=\sum_{k=0}^{\infty} \frac{(-t)^{\alpha k}}{\Gamma(\alpha k+1)}$.

The integral equation equivalent to the boundary value problem (133) is 


$$
y(t)=\int_{a}^{b} G(t, s) R(t, y(s)) d s
$$

where

$$
G(t, s)= \begin{cases}\frac{(t-a)(b-s)}{b-a}, & a \leq t \leq s \leq b \\ \frac{(t-a)(b-s)}{b-a}-(t-s), & a \leq s \leq t \leq b\end{cases}
$$

and

$$
R(t, y(t))=\frac{1-\beta}{B(\beta)} q(t) y(t)+\frac{\beta}{B(\beta)}\left({ }_{a} I^{\beta} q(\cdot) y(\cdot)\right)(t), \quad \beta=\alpha-2
$$

The Green's function $G(t, s)$ defined above has the following properties:

(i) $G(t, s) \geq 0$ for all $a \leq t, s \leq b$;

(ii) $\max _{t \in[a, b]} G(t, s)=G(s, s)$ for $s \in[a, b]$;

(iii) $G(s, s)$ has a unique maximum, given by

$$
\max _{s \in[a, b]} G(s, s)=G\left(\frac{a+b}{2}, \frac{a+b}{2}\right)=\frac{b-a}{4} .
$$

The Lyapunov inequality for the problem (133) is given in the following result.

Theorem 42. If the boundary value problem (133) has a nontrivial solution, where $q$ is a real-valued continuous function on $[a, b]$, then

$$
\int_{a}^{b}\left[\frac{3-\alpha}{B(\alpha-2)}|q(t)|+\frac{\alpha-2}{B(\alpha-2)}\left({ }_{a} I^{\alpha-2}|q(\cdot)|\right)(t)\right] d s>\frac{4}{b-a} .
$$

Remark 1. For $\alpha \rightarrow 2+$, notice that $\frac{3-\alpha}{B(\alpha-2)}|q(t)|+\frac{\alpha-2}{B(\alpha-2)}\left({ }_{a} I^{\alpha-2}|q(\cdot)|\right)(t) \rightarrow$ $|q(t)|$ and hence the inequality (134) reduces to the classical Lyapunov inequality (6).

\section{Lyapunov inequality for discrete fractional boundary value problems}

Let us begin this section with the definitions of integral and derivative of arbitrary order for a function defined on a discrete set. For details, see [56].

The power function is defined by

$$
x^{(y)}=\frac{\Gamma(x+1)}{\Gamma(x+1-y)}, \text { for } x, x-y \in \mathbb{R} \backslash\{\ldots,-2,-1\} .
$$

For $a \in \mathbb{R}$, we define the set $\mathbb{N}_{a}=\{a, a+1, a+2, \ldots\}$. Also, we use the notation $\sigma(s)=s+1$ for the shift operator and $(\Delta f)(t)=f(t+1)-f(t)$ for the forward difference operator. Notice that $\left(\Delta^{2} f\right)(t)=(\Delta \Delta f)(t)$. 
For a function $f: \mathbb{N}_{a} \rightarrow \mathbb{R}$, the discrete fractional sum of order $\alpha \geq 0$ is defined as

$$
\begin{aligned}
\left({ }_{a} \Delta^{0} f\right)(t) & =f(t), t \in \mathbb{N}_{a}, \\
\left({ }_{a} \Delta^{-\alpha} f\right)(t) & =\frac{1}{\Gamma(\alpha)} \sum_{s=a}^{t-\alpha}(t-\sigma(s))^{(\alpha-1)}, f(s), t \in \mathbb{N} a+\alpha, \alpha>0 .
\end{aligned}
$$

The discrete fractional derivative of order $\alpha \in(1,2]$ is defined by

$$
\left({ }_{a} \Delta^{\alpha} f\right)(t)=\left(\Delta^{2}{ }_{a} \Delta^{-(2-\alpha)} f\right)(t), t \in \mathbb{N}_{a+2-\alpha} .
$$

In 2015, Ferreira [57] studied the following conjugate boundary value problem

$$
\left\{\begin{array}{l}
\left(\Delta^{\alpha} y\right)(t)+q(t+\alpha-1) y(t+\alpha-1)=0, t \in[0, b+1]_{\mathbb{N}_{0}} \\
y(\alpha-2)=0=y(\alpha+b+1)
\end{array}\right.
$$

The function $y$ is a solution of the boundary value problem (135) if and only if $y$ satisfies the integral equation

$$
y(t)=\sum_{0}^{b+1} G(t, s) q(s+\alpha-1) f(y(s+\alpha-1)),
$$

where $G(t, s)$ is the Green's function defined by

$$
G(t, s)=\frac{1}{\Gamma(\alpha)} \begin{cases}\frac{t^{(\alpha-1)}(\alpha+b-\sigma(s))^{(\alpha-1)}}{(\alpha+b+1)^{(\alpha-1)}} & \\ -(t-\sigma(s))^{(\alpha-1)}, & s<t-\alpha+1<b+1, \\ \frac{t^{(\alpha-1)}(\alpha+b-\sigma(s))^{(\alpha-1)}}{(\alpha+b+1)^{(\alpha-1)}}, & t-\alpha+1 \leq s \leq b+1,\end{cases}
$$

and that

$$
\begin{gathered}
\max _{s \in[0, b+1]_{\mathbb{N}_{0}}} G(s, s)=G\left(\frac{b}{2}+\alpha-1, \frac{b}{2}\right), \text { if } b \text { is even, } \\
\max _{s \in[0, b+1]_{\mathbb{N}_{0}}} G(s, s)=G\left(\frac{b+1}{2}+\alpha-1, \frac{b+1}{2}\right), \text { if } b \text { is odd, }
\end{gathered}
$$

The Lyapunov inequality for the problem (135) is as follows.

Theorem 43. If the discrete fractional boundary value problem (135) has a nontrivial solution, then

$$
\sum_{s=0}^{b+1}|q(s+\alpha-1)|>4 \Gamma(\alpha) \frac{\Gamma(b+\alpha+2) \Gamma^{2}\left(\frac{b}{2}+2\right)}{(b+2 \alpha)(b+2) \Gamma^{2}\left(\frac{b}{2}+\alpha\right) \Gamma(b+3)}, \text { if } b \text { is even }
$$




$$
\sum_{s=0}^{b+1}|q(s+\alpha-1)|>4 \Gamma(\alpha) \frac{\Gamma(b+\alpha+2) \Gamma^{2}\left(\frac{b+3}{2}\right)}{\Gamma(b+3)\left(\Gamma^{2}\left(\frac{b+1}{2}+\alpha\right)\right.}, \text { if } b \text { is odd. }
$$

As a simple application, consider the right-focal boundary value problem in Theorem 43 with $q=\lambda \in \mathbb{R}$. Then an eigenvalue of the boundary value problem

$$
\left\{\begin{array}{l}
\left(\Delta^{\alpha} y\right)(t)+\lambda y(t+\alpha-1)=0, \quad t \in[0, b+1]_{\mathbb{N}_{0}}, \\
y(\alpha-2)=0=\Delta y(\alpha+b),
\end{array}\right.
$$

must necessarily satisfy the following inequality

$$
|\lambda| \geq \frac{1}{\Gamma(\alpha-1)(b+2)^{2}} .
$$

Ferreira [57] also studied the following right-focal boundary value problem

$$
\left\{\begin{array}{l}
\left(\Delta^{\alpha} y\right)(t)+q(t+\alpha-1) y(t+\alpha-1)=0, t \in[0, b+1]_{\mathbb{N}_{0}}, \\
y(\alpha-2)=0=\Delta y(\alpha+b) .
\end{array}\right.
$$

The function $y$ is a solution of the boundary value problem (139) if and only if $y$ satisfies the integral equation

$$
y(t)=\int_{a}^{b} G(t, s) q(s) y(s) d s,
$$

where $G(t, s)$ is the Green's function defined by

$$
G(t, s)=\frac{1}{\Gamma(\alpha)} \begin{cases}\frac{\Gamma(b+3) t^{(\alpha-1)}\left(\alpha+b-\sigma(s)^{(\alpha-2)}\right.}{\Gamma(\alpha+b+1)} & s<t-\alpha+1<b+1, \\ -(t-\sigma(s))^{(\alpha-1)}, & t-\alpha+1 \leq s \leq b+1, \\ \frac{\Gamma(b+3) t^{(\alpha-1)}\left(\alpha+b-\sigma(s)^{(\alpha-2)}\right.}{\Gamma(\alpha+b+1)}, & \end{cases}
$$

with

$$
\max _{s \in[0, b+1]_{\mathbb{N}_{0}}} G(s+\alpha-1, s)=G(b+\alpha, b+1)=\Gamma(\alpha-1)(b+2) .
$$

The Lyapunov inequality for the problem (139) is presented as follows.

Theorem 44. If the discrete fractional boundary value problem (139) has a nontrivial solution, then

$$
\sum_{s=0}^{b+1}|q(s+\alpha-1)|>\frac{1}{\Gamma(\alpha-1)(b+2)} .
$$

In 2017, Chidouh and Torres [58], studied the following conjugate boundary value problem 


$$
\left\{\begin{array}{l}
\left(\Delta^{\alpha} y\right)(t)+q(t+\alpha-1) f(y(t+\alpha-1))=0, t \in[0, b+1]_{\mathbb{N}_{0}}, \\
y(\alpha-2)=0=y(\alpha+b+1)
\end{array}\right.
$$

where $f \in C\left(\mathbb{R}^{+}, \mathbb{R}^{+}\right)$is nondecreasing and $q:[\alpha-1, \alpha+b]_{\mathbb{N}_{\alpha-1}} \rightarrow \mathbb{R}^{+}$is a nontrivial function.

The function $y$ is a solution of the boundary value problem (142) if and only if $y$ satisfies the integral equation

$$
y(t)=\sum_{0}^{b+1} G(t, s) q(s+\alpha-1) f(y(s+\alpha-1)),
$$

where $G(t, s)$ is the Green's function defined by

$$
G(t, s)=\frac{1}{\Gamma(\alpha)} \begin{cases}\frac{t^{(\alpha-1)}(\alpha+b-s)^{(\alpha-1)}}{(\alpha+b+1)^{(\alpha-1)}}-(t-s-1)^{(\alpha-1)}, & s<t-\alpha+1<b+1, \\ \frac{t^{(\alpha-1)}(\alpha+b-s)^{(\alpha-1)}}{(\alpha+b+1)^{(\alpha-1)}}, & t-\alpha+1 \leq s \leq b+1 .\end{cases}
$$

Moreover, the function $G$ satisfies the following properties:

(i) $G(t, s)>0$ for all $t \in[\alpha-1, \alpha+b]_{\mathbb{N}_{0}}$ and $s \in[1, b+1]_{\mathbb{N}_{1}}$;

(ii) $\max _{[\alpha-1, \alpha+b]_{\mathbb{N}_{0}}} G(t, s)=G(s+\alpha-1, s), s \in[1, b+1]_{\mathbb{N}_{1}}$;

(iii) $G(s+\alpha-1)$ has a unique maximum given by

$$
\max _{s \in[1, b+1]_{\mathbb{N}_{1}}} G(s+\alpha-1)= \begin{cases}\frac{1}{4} \frac{(b+2 \alpha)(b+2) \Gamma^{2}\left(\frac{b}{2}+\alpha\right) \Gamma(b+3)}{\Gamma(\alpha) \Gamma(b+\alpha+2) \Gamma^{2}\left(\frac{b}{2}+\alpha\right)} & \text { if } b \text { is even, } \\ \frac{1}{\Gamma(\alpha)} \frac{(b+3) \Gamma^{2}\left(\frac{b+1}{2}+\alpha\right)}{\Gamma(b+\alpha+2) \Gamma^{2}\left(\frac{b+3}{2}\right)} & \text { if } b \text { is odd. }\end{cases}
$$

The Lyapunov inequality for the problem (142) is expressed as follows.

Theorem 45. If the discrete fractional boundary value problem (142) has a nontrivial solution, then

$$
\sum_{s=0}^{b+1}|q(s+\alpha-1)|>4 \Gamma(\alpha) \frac{\Gamma(b+\alpha+2) \Gamma^{2}\left(\frac{b}{2}+2\right) \eta}{(b+2 \alpha)(b+2) \Gamma^{2}\left(\frac{b}{2}+\alpha\right) \Gamma(b+3) f(\eta)}, \text { if } b \text { is even }
$$

and

$$
\sum_{s=0}^{b+1}|q(s+\alpha-1)|>4 \Gamma(\alpha) \frac{\Gamma(b+\alpha+2) \Gamma^{2}\left(\frac{b+3}{2}\right) \eta}{\Gamma(b+3)\left(\Gamma^{2}\left(\frac{b+1}{2}+\alpha\right) f(\eta)\right.}, \text { if } b \text { is odd }
$$

where $\eta=\max _{[\alpha-1, \alpha+b]_{\mathbb{N}_{\alpha-1}}} y(s+\alpha-1)$. 
In 2017, Ghanbari and Gholami [59] presented the Lyapunov-type inequalities for two special classes of Sturm-Liouville problems equipped with fractional $\Delta$ difference operators, which are given in the next two results.

Theorem 46. Assume that $p:[a, b]_{\mathbb{N}_{0}} \rightarrow \mathbb{R}^{+}$and $q:[\alpha+a-1, \alpha+b-1]_{\mathbb{N}_{\alpha-1}} \rightarrow \mathbb{R}$ are real-valued functions. If $y$ defined on $[\alpha+a-1, \alpha+b-1]_{\mathbb{N}_{\alpha-1}}$ is a nontrivial solution to the fractional Sturm-Liouville problem

$$
\left\{\begin{array}{l}
\Delta_{b-}^{\alpha}\left(p(t) \Delta_{a+}^{\alpha} y(t)\right)+[q(t+\alpha-1)-\lambda] y(t+\alpha-1)=0, \quad t \in(a, b), \\
y(\alpha+a-1)=0, y(\alpha+b)=0,
\end{array}\right.
$$

where $\alpha \in(1 / 2,1)$ and $t=a, a+1, \ldots, b, a, b \in \mathbb{Z}, \lambda \in \mathbb{R}$ such that $a \geq 1, b \geq 3$, then the following Lyapunov type inequality holds:

$$
\sum_{s=a}^{b} \sum_{w=a}^{b}\left(\frac{|q(w+\alpha-1)-\lambda|}{p(s)}\right) \geq \frac{1}{2}
$$

Theorem 47. Suppose that $q:[\alpha+a-1, \alpha+b-1]_{\mathbb{N}_{\alpha-1}} \rightarrow \mathbb{R}$ is a real-valued function for $1<\alpha \leq 2$. Assume that $y$ defined on $[\alpha+a-2, \alpha+b+1]_{\mathbb{N}_{\alpha-2}}$ is a nontrivial solution to the fractional $\Delta$-difference boundary value problem:

$$
\left\{\begin{array}{l}
\Delta_{a+}^{\alpha} y(t)+[q(t+\alpha-1)-\lambda] y(t+\alpha-1)=0, \quad t \in(a, b), \\
y(\alpha+a-2)=0, y(\alpha+b+1)=0,
\end{array}\right.
$$

where $t=a, a+1, \ldots, b, b+1 a, b \in \mathbb{Z}, \lambda \in \mathbb{R}$ such that $a \geq 1, b \geq 2$, then the following Lyapunov-type inequalities hold:

$$
\sum_{a}^{b+1}|q(s+\alpha-1)-\lambda| \geq \Gamma(\alpha) \frac{b-a+2}{b-a+2 \alpha} \frac{\Gamma(\alpha+b-a+2) \Gamma^{2}\left(\frac{b-a}{2}+1\right)}{\Gamma(b-a+2) \Gamma^{2}\left(\frac{b-a}{2}+\alpha\right)},
$$

if $a+b$ is even and

$$
\sum_{a}^{b+1}|q(s+\alpha-1)-\lambda| \geq \Gamma(\alpha) \frac{b-a+3}{b-a+2 \alpha+1} \frac{\Gamma(\alpha+b-a+2) \Gamma^{2}\left(\frac{b-a+1}{2}+1\right)}{\Gamma(b-a+2) \Gamma^{2}\left(\frac{b-a+1}{2}+\alpha\right)},
$$

if $a+b$ is odd.

\section{Lyapunov-type inequality for fractional impulsive boundary value problems}

In 2017, Kayar [60] considered the following impulsive fractional boundary value problem 


$$
\left\{\begin{array}{l}
\left({ }^{C} D^{\alpha} y\right)(t)+q(t) y(t)=0, \quad t \neq \tau_{i}, a<t<b, 1<\alpha<2, \\
\left.\Delta y\right|_{t=\tau_{i}}:=y\left(\tau_{i}^{+}\right)-y\left(\tau_{i}^{-}\right), \quad i=1,2, \ldots, p, \\
\Delta y_{t=\tau_{i}}^{\prime}=-\frac{\gamma_{i}}{\beta_{i}} y\left(\tau_{i}^{-}\right), \quad i=1,2, \ldots, p, \\
y(a)=y(b)=0
\end{array}\right.
$$

where ${ }^{C} D^{\alpha}$ is the Caputo fractional derivative of order $\alpha(1<\alpha \leq 2), q: P L C[a, b] \rightarrow$ $\mathbb{R}$ is a continuous function, $a=\tau_{0}<\tau_{1}<\ldots<\tau_{p}<\tau_{p+1}=b, P L C[a, b]=\{y$ : $[a, b] \rightarrow \mathbb{R}$ is continuous on each interval $\left(\tau_{i}, \tau_{i+1}\right)$, the limits $y\left(\tau_{i}^{ \pm}\right)$exist and $y\left(\tau_{i}^{-}\right)=y\left(\tau_{i}\right)$ for $\left.i=1,2, \ldots, p\right\}$, and $P L C^{1}[a, b]=\left\{y:[a, b] \rightarrow \mathbb{R}, y^{\prime} \in P L C[a, b]\right\}$.

$y \in P L C^{1}([a, b], \mathbb{R})$ is a solution of the boundary value problem 147 if and only if $y$ satisfies the following integral equation

$$
y(t)=-\int_{a}^{b} G(t, s) q(s) y(s) d s-\sum_{a \leq \tau_{i}<b} H\left(t, \tau_{i}\right) \frac{\gamma_{i}}{\beta_{i}} y\left(\tau_{i}\right),
$$

where

$$
G(t, s)=\frac{1}{\Gamma(\alpha)} \begin{cases}\frac{a-t}{b-a}(b-s)^{\alpha-1} & a \leq t \leq s \leq b, \\ \frac{a-t}{b-a}(b-s)^{\alpha-1}-(t-s)^{\alpha-1}, & a \leq s \leq t \leq b,\end{cases}
$$

and

$$
H\left(t, \tau_{i}\right)=\frac{1}{\Gamma(\alpha)} \begin{cases}\frac{a-t}{b-a}\left(b-\tau_{i}\right) & a \leq t \leq \tau_{i} \leq b, \\ \frac{a-\tau_{i}}{b-a}(b-t), & a \leq \tau_{i} \leq t \leq b .\end{cases}
$$

Furthermore, the functions $G$ and $H$ satisfy the following properties:

(i) $G(t, s) \mid \leq \frac{1}{\Gamma(\alpha)} \frac{(\alpha-1)^{\alpha-1}}{\alpha^{\alpha}}(b-a)^{\alpha-1}$, for all $a \leq t, s \leq b$;

(ii) $H\left(t, \tau_{i}\right) \leq 0$ and $\left|H\left(t, \tau_{i}\right)\right| \leq \frac{b-a}{4}$, for all $a \leq t, \tau_{i} \leq b$.

The Lyapunov inequality for the problem (147) is the following.

Theorem 48. (Lyapunov inequality) If the problem (147) has a nontrivial solution $y(t) \neq 0$ on $(a, b)$, then

$$
\int_{a}^{b}|q(s)| d s+\sum_{a \leq \tau_{i}<b}\left(\frac{\gamma_{i}}{\beta_{i}}\right)^{+}>\min \left\{\frac{4}{b-a}, \frac{\Gamma(\alpha) \alpha^{\alpha}}{[(\alpha-1)(b-a)]^{\alpha-1}}\right\},
$$

where $\left(\frac{\gamma_{i}}{\beta_{i}}\right)^{+}=\max \left\{\frac{\gamma_{i}}{\beta_{i}}, 0\right\}$. 


\section{Lyapunov inequality for boundary value problems involving Hilfer fractional derivative}

A generalization of both Riemann-Liouville and Caputo derivatives, known as generalized Riemann-Liouville derivative of order $\alpha \in(0,1)$ and type $\beta \in[0,1]$, was proposed by R. Hilfer in [61]. Such a derivative interpolates between the RiemannLiouville and Caputo derivative in some sense. For properties and applications of the Hilfer derivative, see [62, 63] and references cited therein.

Definition 8. The generalized Riemann-Liouville fractional derivative or Hilfer fractional derivative of order $\alpha$ and parameter $\beta$ for a function $u$ is defined as

$$
{ }^{H} D^{\alpha, \beta} u(t)=I^{\beta(n-\alpha)} D^{n} I^{(1-\beta)(n-\alpha)} u(t)
$$

where $n-1<\alpha<n, \quad 0 \leq \beta \leq 1, t>a, \quad D=\frac{d}{d t}$.

Remark 2. The Hilfer fractional derivative corresponds to the Riemann-Liouville fractional derivative for $\beta=0$, that is, ${ }^{H} D^{\alpha, 0} u(t)=D^{n} I^{n-\alpha} u(t)$, while it corresponds to the Caputo fractional derivative for $\beta=1$ given by ${ }^{H} D^{\alpha, 1} u(t)=$ $I^{n-\alpha} D^{n} u(t)$.

In 2016, Pathak [69] studied Lyapunov-type inequalities for fractional boundary value problems involving Hilfer fractional derivative and Dirichlet, mixed Dirichlet and Neumann boundary conditions.

Let us first consider the Dirichlet boundary value problem given by

$$
\left\{\begin{array}{l}
\left(D^{\alpha, \beta} y\right)(t)+g(t) y(t)=0, \quad t \in(a, b), 1<\alpha \leq 2,0 \leq \beta \leq 1 \\
y(a)=y(b)=0
\end{array}\right.
$$

which is equivalent to the integral equation

$$
y(t)=\int_{a}^{b} G(t, s) q(s) y(s) d s
$$

where

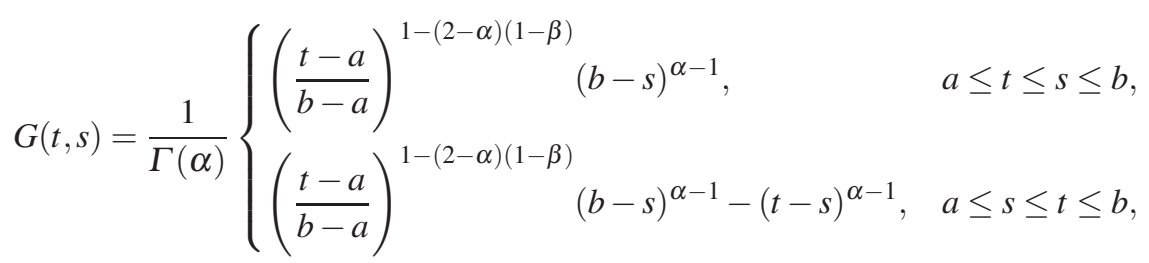

is the Green's function satisfying the property:

$$
|G(t, s)| \leq \frac{(b-a)^{\alpha-1}[\alpha-1+\beta(2-\alpha)]^{\alpha-1+\beta(2-\alpha)}[\alpha-1]^{\alpha-1}}{\Gamma(\alpha)[\alpha-(2-\alpha)(1-\beta)]^{\alpha-(2-\alpha)(1-\beta)}},(t, s) \in[a, b] \times[a, b]
$$


Theorem 49. (Lyapunov-type inequality) If a nontrivial continuous solution of the problem (148) exists, then

$$
\int_{a}^{b}|q(s)| d s \geq \frac{\Gamma(\alpha)[\alpha-(2-\alpha)(1-\beta)]^{\alpha-(2-\alpha)(1-\beta)}}{(b-a)^{\alpha-1}[\alpha-1+\beta(2-\alpha)]^{\alpha-1+\beta(2-\alpha)}(\alpha-1)^{\alpha-1}} .
$$

Next we consider a fractional boundary value problems involving Hilfer fractional derivative and mixed Dirichlet and Neumann boundary conditions:

$$
\left\{\begin{array}{l}
\left(D^{\alpha, \beta} y\right)(t)+q(t) y(t)=0, \quad t \in(a, b), 1<\alpha \leq 2,0 \leq \beta \leq 1 \\
y(a)=y^{\prime}(b)=0
\end{array}\right.
$$

which is equivalent to the integral equation

$$
y(t)=\int_{a}^{b} G(t, s) q(s) y(s) d s,
$$

where $G(t, s)=\frac{H(t, s)}{\Gamma(\alpha)(b-s)^{2-\alpha}}$ and

$$
H(t, s)= \begin{cases}\frac{(\alpha-1)(t-a)^{1-(2-\alpha)(1-\beta)}(b-a)^{(2-\alpha)(1-\beta)}}{1-(2-\alpha)(1-\beta)}, & a \leq t \leq s \leq b, \\ \frac{(\alpha-1)(t-a)^{1-(2-\alpha)(1-\beta)}(b-a)^{(2-\alpha)(1-\beta)}}{1-(2-\alpha)(1-\beta)} & \\ -(t-s)^{\alpha-1}(b-s)^{2-\alpha}, & a \leq s \leq t \leq b .\end{cases}
$$

The function $H$ satisfies the following property:

$$
|H(t, s)| \leq \frac{b-a}{\alpha-1+\beta(2-\alpha)} \max \{\alpha-1, \beta(2-\alpha)\},(t, s) \in[a, b] \times[a, b] .
$$

Theorem 50. (Lyapunov-type inequality) If a nontrivial continuous solution of the problem (149) exists, then

$$
\int_{a}^{b}(b-s)^{\alpha-2}|q(s)| d s \geq \frac{\Gamma(\alpha)(\alpha-1+\beta(2-\alpha))}{(b-a) \max \{\alpha-1, \beta(2-\alpha)\}} .
$$

Now we establish a Lyapunov-type inequality for another fractional boundary value problems with Hilfer fractional derivative and mixed Dirichlet and Neumann boundary conditions:

$$
\left\{\begin{array}{l}
\left(D^{\alpha, \beta} y\right)(t)+q(t) y(t)=0, \quad t \in(a, b), 1<\alpha \leq 2,0 \leq \beta \leq 1 \\
y(a)=y^{\prime}(a)=y^{\prime}(b)=0
\end{array}\right.
$$

which can be transformed to the integral equation: 


$$
y(t)=\int_{a}^{b} G(t, s) q(s) y(s) d s
$$

where

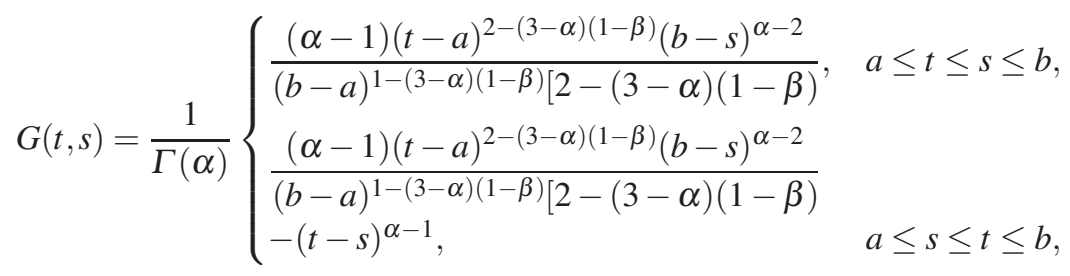

is the Green's function satisfying the property:

$$
|G(t, s)| \leq \frac{2(b-a)^{\alpha-1}(\alpha-2)^{\alpha-2}}{\Gamma(\alpha)[2-(3-\alpha)(1-\beta)]^{\alpha-1}},(t, s) \in[a, b] \times[a, b] .
$$

Theorem 51. (Lyapunov-type inequality) If a nontrivial continuous solution of the problem (150) exists, then

$$
\int_{a}^{b}|q(s)| d s \geq \frac{\Gamma(\alpha)[2-(3-\alpha)(1-\beta)]^{\alpha-1}}{(b-a)^{\alpha-1}(\alpha-2)^{\alpha-2}} .
$$

Finally we consider the following fractional boundary value problem with Hilfer fractional derivative and a mixed set of fractional Dirichlet, Neumann and fractional Neumann boundary conditions

$$
\left\{\begin{array}{l}
\left(D^{\alpha, \beta} y\right)(t)+q(t) y(t)=0, \quad a<t<b, 2<\alpha \leq 3,0 \leq \beta \leq 1 \\
\left(I^{(3-\alpha)(1-\beta)} y\right)(a)=0, y^{\prime}(b)=0, \frac{d^{2}}{d t^{2}}\left(I^{(3-\alpha)(1-\beta)} y\right)(a)=0
\end{array}\right.
$$

The integral equation equivalent to the Problem (151) is

$$
y(t)=\int_{a}^{b} G(t, s) q(s) y(s) d s,
$$

where

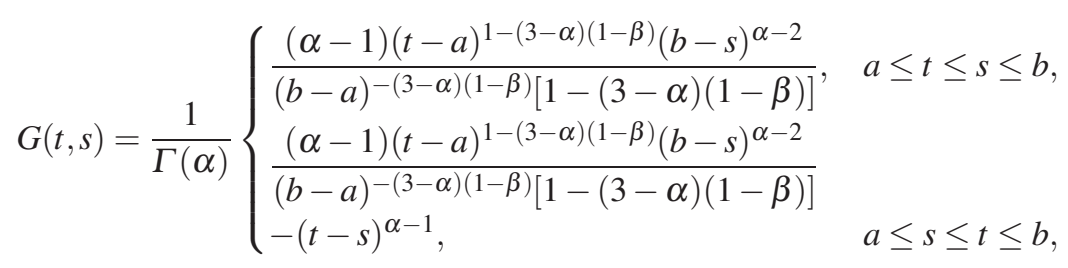

such that 


$$
|G(t, s)| \leq \frac{(\alpha-2)^{\alpha-2}(b-a)^{\alpha-1}}{\Gamma(\alpha)[1-(3-\alpha)(1-\beta)]^{\alpha-1}}, \quad(t, s) \in[a, b] \times[a, b] .
$$

Theorem 52. (Lyapunov inequality) If a nontrivial continuous solution of the problem (151) exists, then

$$
\int_{a}^{b}|q(s)| d s \geq \frac{\Gamma(\alpha)[1-(3-\alpha)(1-\beta)]^{\alpha-1}}{(b-a)^{\alpha-1}(\alpha-2)^{\alpha-2}} .
$$

In 2017, Kirane and Torebek [64] obtained Lyapunov-type inequalities for the following fractional boundary value problem

$$
\left\{\begin{array}{l}
D_{a}^{\alpha, \gamma} y(t)+q(t) f(y(t))=0, \quad a<t<b, 1<\alpha \leq \gamma<2, \\
y(a)=y(b)=0
\end{array}\right.
$$

where $D_{a}^{\alpha, \gamma}$ is a generalized Hilfer fractional derivative of order $\alpha \in \mathbb{R}(m-1<$ $\alpha<m, m \in \mathbb{N}$ ) and type $\gamma$, defined as

$$
D_{a}^{\alpha, \gamma} f(t)=I_{a}^{\gamma-\alpha} \frac{d^{m}}{d t^{m}} I_{a}^{m-\gamma} f(t),
$$

and $q:[a, b] \rightarrow \mathbb{R}$ is a nontrivial Lebesgue integrable function.

The integral representation for the solution of the boundary value problem (152) is

$$
y(t)=\int_{a}^{b} G(t, s) q(s) f(y(s)) d s,
$$

where $G(t, s)$ is the Green's function given by

$$
G(t, s)= \begin{cases}\left(\frac{t-a}{b-a}\right)^{\gamma-1} \frac{(b-s)^{\alpha-1}}{\Gamma(\alpha)}-\frac{(t-s)^{\alpha-1}}{\Gamma(\alpha)}, & a \leq s \leq t \leq b, \\ \left(\frac{t-a}{b-a}\right)^{\gamma-1} \frac{(b-s)^{\alpha-1}}{\Gamma(\alpha)}, & a \leq t \leq s \leq b .\end{cases}
$$

Further, the above Green's function $G(t, s)$ satisfies the following properties:

(i) $G(t, s) \geq 0$ for $a \leq t, s \leq b$;

(ii) $\max _{a \leq t \leq b} G(t, s)=G(s, s), s \in[a, b]$;

(iii) $G(s, s)$ has a unique maximum, given gy

$$
\max _{a \leq s \leq b} G(s, s)=\frac{(\alpha-1)^{\alpha-1}}{(\gamma+\alpha-2)^{\gamma+\alpha-2}} \frac{((\gamma-1) b-(\alpha-1) a)^{\gamma-1}}{\Gamma(\alpha)(b-a)^{\gamma-\alpha}} .
$$

They obtained the following Lyapunov-type inequalities.

Theorem 53. If the fractional boundary value problem (152) has a nontrivial solution for a real valued continuous function $q$, then 


$$
\int_{a}^{b}|q(s)| d s>\frac{(\gamma+\alpha-2)^{\gamma+\alpha-2}}{(\alpha-1)^{\alpha-1}} \frac{\Gamma(\alpha)(b-a)^{\gamma-\alpha}}{((\gamma-1) b-(\alpha-1) a)^{\gamma-1}} .
$$

Theorem 54. Let $q:[a, b] \rightarrow \mathbb{R}$ be a real notrivial Lebesgue integrable function and $f \in C\left(\mathbb{R}_{+}, \mathbb{R}_{+}\right)$be a concave and nondecreasing function. If there exists a nontrivial solution y for the problem (152), then

$$
\int_{a}^{b}|q(s)| d s>\frac{(\gamma+\alpha-2)^{\gamma+\alpha-2}}{(\alpha-1)^{\alpha-1}} \frac{\Gamma(\alpha)(b-a)^{\gamma-\alpha}}{((\gamma-1) b-(\alpha-1) a)^{\gamma-1}} \frac{\omega}{f(\omega)}
$$

where $\omega=\max _{t \in[a, b]} y(t)$.

Theorem 55. (Hartman-Wintner type inequality) Let the functions $q$ and $f$ satisfy the conditions of Theorem 54. Suppose that the fractional boundary value problem (152) has a nontrivial solution. Then

$$
\int_{a}^{b}(s-a)^{\gamma-1}(b-s)^{\alpha-1} q^{+}(s) d s>\frac{\|y\|}{f(\|y\|)} \Gamma(\alpha)(b-a)^{\gamma-1} .
$$

Corollary 20. If $f(y)=y$ (linear case) and $q \in L^{1}\left([a, b], \mathbb{R}_{+}\right)$, then

$$
\int_{a}^{b}(s-a)^{\gamma-1}(b-s)^{\alpha-1} q^{+}(s) d s>\Gamma(\alpha)(b-a)^{\gamma-1} .
$$

\section{Lyapunov-type inequality with the Katugampola fractional derivative}

In 2018, Lupinska and Odzijewicz [65] obtained a Lyapunov-type inequality for the following fractional boundary value problem

$$
\left\{\begin{array}{l}
D_{a+}^{\alpha, \rho} y(t)+q(t) y(t)=0, \quad a<t<b, \alpha>0, \rho>0 \\
y(a)=y(b)=0
\end{array}\right.
$$

where $D_{a+}^{\alpha, \rho}$ is the Katugampola fractional derivative of order $\alpha$, defined as

$$
D_{a+}^{\alpha, \gamma} f(t)=\left(t^{1-\alpha} \frac{d}{d t}\right)^{n} I_{a+}^{n-\alpha} f(t)
$$

for $t \in(a, b), n=[\alpha]+1,0<a<t<b \leq \infty$ and $q:[a, b] \rightarrow \mathbb{R}$ is a continuous function. Here $I_{a+}^{\alpha, \rho}$ is the Katugampola fractional integral defined by

$$
I_{a+}^{\alpha, \rho} f(t)=\frac{\rho^{1-\alpha}}{\Gamma(\alpha)} \int_{a}^{t} \frac{s^{\rho-1}}{\left(t^{\rho}-s^{\rho}\right)^{1-\alpha}} f(s) d s
$$


The integral representation for the solution of the boundary value problem (153) is

$$
y(t)=\int_{a}^{b} G(t, s) q(s) y(s) d s,
$$

where $G(t, s)$ is the Green's function given by

$$
G(t, s)=\frac{\rho^{1-\alpha}}{\Gamma(\alpha)} \begin{cases}\frac{s^{\rho-1}}{\left(b^{\rho}-s^{\rho}\right)^{1-\alpha}}\left(\frac{t^{\rho}-a^{\rho}}{b^{\rho}-a^{\rho}}\right)^{\alpha-1}, & a \leq t \leq s \leq b, \\ \frac{s^{\rho-1}}{\left(b^{\rho}-s^{\rho}\right)^{1-\alpha}}\left(\frac{t^{\rho}-a^{\rho}}{b^{\rho}-a^{\rho}}\right)^{\alpha-1}-\frac{s^{\rho-1}}{\left(t^{\rho}-s^{\rho}\right)^{1-\alpha}}, & a \leq s \leq t \leq b,\end{cases}
$$

which satisfies the following properties:

(i) $G(t, s) \geq 0$ for $a \leq t, s \leq b$;

(ii) $\max _{a \leq t \leq b} G(t, s)=G(s, s) \leq \frac{\max \left\{a^{\rho-1}, b^{\rho-1}\right\}}{\Gamma(\alpha)}\left(\frac{b^{\rho}-a^{\rho}}{4 \rho}\right)^{\alpha-1}, s \in[a, b]$.

They obtained the following Lyapunov-type inequality.

Theorem 56. If the fractional boundary value problem (153) has a nontrivial solution for a real valued continuous function $q$, then

$$
\int_{a}^{b}|q(s)| d s>\frac{\Gamma(\alpha)}{\max \left\{a^{\rho-1}, b^{\rho-1}\right\}}\left(\frac{4 \rho}{b^{\rho}-a^{\rho}}\right)^{\alpha-1} .
$$

Remark 3. In the special case when $\rho=1$ in Theorem 56, we get the following result

$$
\int_{a}^{b}|q(s)| d s \geq \Gamma(\alpha)\left(\frac{4}{b-a}\right)^{\alpha-1}
$$

which is Theorem 2, while taking $\rho \rightarrow 0^{+}$in Theorem 56, we have the Lyapunov's type inequality for the Hadamard fractional derivative:

$$
\int_{a}^{b}|q(s)| d s \geq \alpha \Gamma(\alpha)\left(\frac{\log (b / a)}{4}\right)^{1-\alpha}
$$

\section{Lyapunov inequality for a boundary value problem involving the conformable derivative}

Recently, Khalil et al. [66] introduced a new derivative, which appears in the form of a limit like the classical derivative and is known as the conformable derivative. Later, this new local derivative was improved by Abdeljawad [67]. The importance of the conformable derivative is that it has properties similar to the ones of the classical derivative. However, the conformable derivative does not satisfy the index law [68, 70] and the zero order derivative property, that is, the zero order derivative of a differentiable function does not return to the function itself. 
In 2017, Khaldi et al. [71] obtained a Lyapunov-type inequality for the following boundary value problem involving the conformable derivative of order $1<\alpha<2$ and Dirichlet boundary conditions:

$$
\left\{\begin{array}{l}
T_{\alpha} y(t)+q(t) y(t)=0, \quad t \in(a, b), \\
y(a)=y^{\prime}(b)=0
\end{array}\right.
$$

where $T_{\alpha}$ denotes the conformable derivative of order $\alpha$ and $q:[a, b] \rightarrow \mathbb{R}$ is a real continuous function.

The conformable derivative of order $0<\alpha<1$ for a function $g:[a, \infty) \rightarrow \mathbb{R}$ is defined by

$$
T_{\alpha} g(t)=\lim _{\varepsilon \rightarrow 0} \frac{g\left(t+\varepsilon(t-a)^{1-\alpha}\right)-g(t)}{\varepsilon}, t>a .
$$

If $T_{\alpha} g(t)$ exists on $(a, b), b>a$ and $\lim _{t \rightarrow a+} T_{\alpha} g(t)$ exists, then we define $T_{\alpha} g(a)=$ $\lim _{t \rightarrow a+} T_{\alpha} g(t)$.

The conformable derivative of order $n<\alpha<n+1$ of a function $g:[a, \infty) \rightarrow \mathbb{R}$, when $g^{(n)}$ exists, is defined as

$$
T_{\alpha} g(t)=T_{\beta} g^{(n)}(t),
$$

where $\beta=\alpha-n \in(0,1)$.

The solution $y$ of the problem (154) can be written as

$$
y(t)=\int_{a}^{b} G(t, s) q(s) y(s) d s,
$$

where

$$
G(t, s)=\frac{1}{b-a} \begin{cases}(b-s)(t-a), & a \leq t \leq s \leq b, \\ -(b-a)(t-s)+(b-s)(t-a), & a \leq s \leq t \leq b,\end{cases}
$$

is the Green's function, which is nonnegative, continuous and satisfies the property:

$$
0 \leq G(t, s) \leq b-a, \text { for all } t, s \in[a, b] .
$$

Theorem 57. (Lyapunov inequality) Let $q \in C([a, b], \mathbb{R})$. If the boundary value problem (154) has a solution $y \in A C^{2}([a, b], \mathbb{R})$ such that $y(t) \neq 0$ a.e. on $(a, b)$, then

$$
\int_{a}^{b}|q(s)|(s-a)^{\alpha-2} d s \geq \frac{4}{b-a} .
$$

In 2017, Abdeljawad et al. [72] obtained Lyapunov-type inequality for a Dirichlet boundary value problem involving conformable derivative of order $1<\alpha<2$ : 


$$
\left\{\begin{array}{l}
T_{\alpha} y(t)+q(t) y(t)=0, \quad t \in(a, b), \\
y(a)=y(b)=0
\end{array}\right.
$$

where $T_{\alpha}$ denotes the conformable derivative of order $\alpha$ and $q:[a, b] \rightarrow \mathbb{R}$ is a real continuous function.

The solution for the boundary value problem (155) is

$$
y(t)=\int_{a}^{b} G(t, s) q(s) y(s) d s,
$$

where $G(t, s)$ is the Green's function given by

$$
G(t, s)= \begin{cases}\frac{(t-a)(b-s)}{b-a} \cdot(s-a)^{\alpha-2}, & a \leq t \leq s \leq b, \\ \left(\frac{(t-a)(b-s)}{b-a}-(t-s)\right) \cdot(s-a)^{\alpha-2}, & a \leq s \leq t \leq b,\end{cases}
$$

which satisfies the properties:

(i) $G(t, s) \geq 0$ for all $a \leq t, s \leq b$;

(ii) $\max _{t \in[a, b]} G(t, s)=G(s, s)$ for $s \in[a, b]$;

(iii) $G(t, s)$ has a unique maximum, given by

$$
\max _{s \in[a, b]} G(s, s)=G\left(\frac{a+(\alpha-1) b}{\alpha}, \frac{a+(\alpha-1) b}{\alpha}\right)=\frac{(b-a)^{\alpha-1}(\alpha-1)^{\alpha-1}}{\alpha^{\alpha}} .
$$

The Lyapunov inequality for the problem (155) is given in the following result.

Theorem 58. If the problem (155) has a nontrivial solution, where $q$ is a real valued continuous function on $[a, b]$, then

$$
\int_{0}^{1}|q(s)| d s>\frac{\alpha^{\alpha}}{(b-a)^{\alpha-1}(\alpha-1)^{\alpha-1}} .
$$

\section{References}

1. Z. Nehari, On the zeros of solutions of second-order linear differential equations, Amer. J. Math. 76 (1954), 689-697.

2. A.M. Lyapunov, Probléme général de la stabilité du mouvement, (French Transl. of a Russian paper dated 1893), Ann. Fac. Sci. Univ. Toulouse 2 (1907), 27-247; Reprinted in: Ann. Math. Studies, No. 17, Princeton (1947).

3. A. Wintner, On the nonexistence of conjugate points, Am. J. Math. 73 (1951), 368-380.

4. P. Hartman, Ordinary Differential Equations, Society for Industrial and Applied Mathematics, 2002.

5. D. Cakmak, Lyapunov-type integral inequalities for certain higher order differential equations, Appl. Math. Comput. 216 (2010), 368-373.

6. S. Clark, D. Hinton, A Lyapunov inequality for linear Hamiltonian systems, Math. Inequal. Appl. 1 (1998), 201-209. 
7. A. Tiryaki, Recent developments of Lyapunov-type inequalities. Adv. Dyn. Syst. Appl. 5 (2010), 231-248.

8. N. Parhi, S. Panigrahi, Lyapunov-type inequality for higher order differential equations, Math. Slovaca 52 (2002) 31-46.

9. X. Yang, On Lyapunov-type inequality for certain higher-order differential equations, Appl. Math. Comput. 134 (2003), 307-317.

10. X. Yang, K. Lo, Lyapunov-type inequality for a class of even-order differential equations, Appl. Math. Comput. 215 (2010), 3884-3890.

11. Q.-M. Zhang, X. H. Tang, Lyapunov inequalities and stability for discrete linear Hamiltonian systems, Appl. Math. Comput. 218 (2011), 574-582.

12. S.S. Cheng, Lyapunov inequalities for differential and difference equations, Fasc. Math. $\mathbf{2 3}$ (1991) 25-41.

13. R.C. Brown, D.B. Hinton, Lyapunov inequalities and their applications, in: Survey on Classical Inequalities. Mathematics and Its Applications 517, Springer, Dordrecht, 2000.

14. A.A. Kilbas, H.M. Srivastava, J.J. Trujillo, Theory and Applications of Fractional Differential Equations 204, Elsevier Science, Amsterdam, 2006.

15. I. Podlubny, Fractional Differential Equations, Academic Press, San Diego, 1999.

16. R.A.C. Ferreira, A Lyapunov-type inequality for a fractional boundary-value problem, Fract. Calc. Appl. Anal. 16 (2013), 978-984.

17. R.A.C. Ferreira, On a Lyapunov-type inequality and the zeros of a certain Mittag-Leffler function, J. Math. Anal. Appl. 412 (2014), 1058-1063.

18. M. Jleli, B. Samet, Lyapunov type inequalities for a fractional differential equations with mixed boundary value problems, Math. Inequal. Appl. 18 (2015), 443-451.

19. M. Jleli, L. Ragoub, B. Samet, A Lyapunov-type inequality for a fractional differential equation under a Robin boundary condition, J. Function Spaces 2015 (2015), 468536 (5 pages).

20. J. Rong, C.Z. Bai, Lyapunov-type inequality for a fractional differential equations with fractional boundary value problems, Adv. Difference Equ. 2015 (2015), 82 (10 pages).

21. M. Jleli, B. Samet, Lyapunov-type inequalities for fractional boundary-value problems, Electron. J. Differential Equations 2015 (2015), 1-11.

22. D. O'Regan, B. Samet, Lyapunov-type inequalities for a class of fractional differential equations, J. Inequal. Appl. 2015 (2015), 247 (10 pages).

23. S. Sitho, S.K. Ntouyas, J. Tariboon, Existence results for hybrid fractional integro-differential equations, Bound. Value Probl. 2015 (2015), 113 (13 pages).

24. M. Al-Qurashi, L. Ragoub, Lyapunov's inequality for a fractional differential equation subject to a non-linear integral condition, ADVCOMP 2016 : The Tenth International Conference on Advanced Engineering Computing and Applications in Sciences, 98-101.

25. R.A. C. Ferreira, Lyapunov-type inequalities for some sequential fractional boundary value problems, Adv. Dynam. Syst. Appl. 11 (2016), 33-43.

26. M. Al-Qurashia, L.Ragoub, Nonexistence of solutions to a fractional differential boundary value problem, J. Nonlinear Sci. Appl. 9 (2016), 2233-2243.

27. S. Dhar, Q. Kong, M. McCabe, Fractional boundary value problems and Lyapunov-type inequalities with fractional integral boundary conditions, Electron. J. Qual. Theory Differ. Equ. 2016 2016, 1-16.

28. S. Dhar, Q. Kong, Lyapunov-type inequalities for $\alpha$-th order fractional differential equations with $2<\alpha \leq 3$ and fractional boundary conditions, Electron. J. Differential Equations 2017 (2017), 1-15.

29. M. Jleli, J. Nieto, B. Samet, Lyapunov-type inequalities for a higher order fractional differential equation with fractional integral boundary conditions, Electron. J. Qual. Theory Differ. Equ. 2017 (2017), 1-17.

30. I. Cabrera, B. Lopez, K. Sadarangani, Lyapunov type inequalities for a fractional two-point boundary value problem, Math. Meth. Appl. Sci. 40 (2017), 3409-3414.

31. X. Wang, Y. Peng, W. Lu, Lyapunov-type inequalities for certain higher order fractional differential equations, J. Nonlinear Sci. Appl. 10 (2017), 5064-5071.

32. R.A.C. Ferreira, A Lyapunov-type inequality for a fractional boundary value problem, Fract. Calc. Appl. Anal. 16 (2013), 978-984. 
33. R.P. Agarwal, A. Zbekler, Lyapunov type inequalities for mixed nonlinear Riemann-Liouville fractional differential equations with a forcing term, J. Comput. Appl. Math. 314 (2017), 6978.

34. L. Zhang, Z. Zheng, Lyapunov type inequalities for the Riemann-Liouville fractional differential equations of higher order, Adv. Difference Equ. 2017 (2017), 270 (20 pages).

35. A. Chidouh, D.F.M. Torres, A generalized Lyapunov's inequality for a fractional boundary value problem, J. Comput. Appl. Math. 312 (2017), 192-197.

36. D. Ma, A generalized Lyapunov inequality for a higher-order fractional boundary value problem, J. Inequal. Appl. 2016 (2016), 261 (11 pages).

37. Y. Ru, F. Wang, T. An, Y. An, Some results on the fractional order Sturm-Liouville problems, Adv. Difference Equ. 2017 (2017), 320 (19 pages).

38. I. Cabrera, J. Rocha, K.B. Sadarangani, Lyapunov type inequalities for a fractional thermostat model, RACSAM 112 (2018), 17-24.

39. P. Guidotti, S. Merino, Gradual loss of positivity and hidden invariant cones in a scalar heat equation, Diff. Integr. Equ. 13 (2000), 1551-1568.

40. I. Cabrera, K. Sadaragani, B. Samet, Hartman-Winter-type inequalities for a class of nonlocal fractional boundary value problems, Math. Methods Appl. Sci. 40 (2017), 129-136.

41. Y. Wang, Q. Wang, Lyapunov-type inequalities for fractional differential equations under multi-point boundary conditions, J. Comput. Anal. Appl. 26 (2019), 707-716.

42. N. Al Arifi, I. Altun, M. Jleli, A. Lashin, B. Samet, Lyapunov-type inequalities for a fractional p-Laplacian equation, J. Inequal. Appl. 2016 (2016), 189 (11 pages).

43. Y. Liu, D. Xie, D. Yang, C. Bai, Two generalized Lyapunov-type inequalities for a fractional p-Laplacian equation with fractional boundary conditions, J. Inequal. Appl. 2017 (2017), 98 (11 pages).

44. A. Guezane-Lakoud, R. Khaldi, D.F.M. Torres, Lyapunov-type inequality for a fractional boundary value problem with natural conditions, D.F.M. SeMA 75 (2018), 157-162.

45. Q. Ma, C. Na, J. Wang, A Lyapunov-type inequality for fractional differential equation with Hadamard derivative, J. Math. Inequal. 11 (2017), 135-141.

46. B. Ahmad, A. Alsaedi, S.K. Ntouyas, J. Tariboon, Hadamard-Type Fractional Differential Equations, Inclusions and Inequalities, Springer, 2017.

47. N.G. Abuj, D.B. Pachpatte, Lyapunov-type inequality for fractional differential equation with $k$-Prabhakar derivative, IJPAM 113 (2017), 563-574.

48. S. Eshaghi, A. Ansari, Lyapunov inequality for fractional differential equations with Prabhakar derivative, Math. Inequal. Appl. 19 (2016), 349-358.

49. D.B. Pachpatte, N.G. Abuj, A.D. Khandagale, Lyapunov-type inequality for hybrid fractional differential equation with Prabhakar derivative, Inter. J. Pure Appl. Math. 113 (2017), 563574.

50. M. Jleli, B. Samet, Lyapunov-type inequality for a fractional $q$-difference boundary value problem, J. Nonlinear Sci. Appl. 9 (2016), 1965-1976.

51. B. Ahmad, S.K. Ntouyas, J. Tariboon, Quantum Calculus: New Concepts, Impulsive IVPs and BVPs, Inequalities, World Scientific, Singapore, 2016.

52. M. Jleli, M. Kirane, B. Samet, Hartman-Wintner-type inequality for a fractional boundary value problem via a fractional derivative with respect to another function, Discrete Dyn. Nat. Soc., 2017 (2017), 5123240 (8 pages).

53. Y. Chen, Y-C. Liou, C-H. Lo, Lyapunov inequality for a class of fractional differential equations with Dirichlet boundary conditions, J. Nonlinear Sci. Appl. 10 (2017), 3357-3363.

54. M. Jleli, M. Kirane, B. Samet, Lyapunov-type inequalities for fractional quasilinear problems via variational methods, J. Nonlinear Sci. Appl. 10 (2017), 2471-2486.

55. T. Abdeljawad, A Lyapunov type inequality for fractional operators with nonsingular MittagLeffler kernel, J. Inequal. Appl. 2017 (2017), 130 (11 pages).

56. Ch. Goodrich, A.C. Peterson, Discrete Fractional Calculus, Springer, 2015.

57. R.A.C. Ferreira, Some discrete fractional Lyapunov-type inequalities, Frac. Differ. Calc. 5 (2015), 87-92. 
58. A. Chidouh, D.F.M. Torres, Existence of positive solutions to a discrete fractional boundary value problem and corresponding Lyapunov-type inequalities, Opuscula Math. 38 (2018), 3140.

59. K. Ghanbari, Y. Gholami, New classes of Lyapunov-type inequalities of fractional $\Delta$ difference Sturm-Liouville problems with applications, Bull. Iranian Math. Soc. 43 (2017), 385-408.

60. Z. Kayar, An existence and uniqueness result for linear fractional impulsive boundary value problems as an application of Lyapunov type inequality, Hacettepe J. Math. Stat. 47 (2018), 287-297.

61. R. Hilfer (Ed.), Applications of Fractional Calculus in Physics, World Scientific, Singapore, 2000.

62. R. Hilfer, Experimental evidence for fractional time evolution in glass forming materials, $J$. Chem. Phys. 284 (2002), 399-408.

63. R. Hilfer, Y. Luchko, Z. Tomovski, Operational method for the solution of fractional differential equations with generalized Riemann-Liouville fractional derivatives, Frac. Calc. Appl. Anal. 12 (2009), 299-318

64. M. Kirane, B. Torebek, Lyapunov and Hartman-Wintner type inequalities for a nonlinear fractional boundary value problem with generalized Hilfer derivative, arXiv:1702.06073 $\mathrm{v}$ [math.CA].

65. B. Lupinska, T. Odzijewicz, A Lyapunov-type inequality with the Katugampola fractional derivative, Math. Meth. Appl. Sci. (2018), 1-12.

66. R. Khalil, M. Al Horani, A. Yousef, M. Sababheh, A new definition of fractional derivative, $J$. Comput. Appl. Math. 264 (2014), 65-70.

67. T. Abdeljawad, On conformable fractional calculus, J. Comput. Appl. Math. 279 (2015), 5766.

68. U.N. Katugampola, Correction to What is a fractional derivative? by Ortigueira and Machado [J. Comput. Phys. 293 (2015), 413] J. Comput. Phys. 321 (2016), 1255-1257.

69. N.S. Pathak, Lyapunov-type inequality for fractional boundary value problems with Hilfer derivative, Math. Inequal. Appl. 21 (2018), 179-200.

70. M.D. Ortigueira, J.A. Tenreiro Machado, What is a fractional derivative? J. Comput. Phys. 293 (2015), 4-13.

71. R. Khaldi, A. Guezane-Lakoud, Lyapunov inequality for a boundary value problem involving conformable derivative, Progr. Fract. Differ. Appl. 3 (2017), 323-329.

72. T. Abdeljawad, J. Alzabut, F. Jarad, A generalized Lyapunov-type inequality in the frame of conformable derivatives, Adv. Difference Equ. 2017 (2017), 321 (10 pages). 